\title{
FREDHOLMNESS OF SINGULAR INTEGRAL OPERATORS WITH PIECEWISE CONTINUOUS COEFFICIENTS ON WEIGHTED BANACH FUNCTION SPACES
}

\author{
ALEXEI YU. KARLOVICH
}

\begin{abstract}
We prove necessary conditions for the Fredholmness of singular integral operators with piecewise continuous coefficients on weighted Banach function spaces. These conditions are formulated in terms of indices of submultiplicative functions associated with local properties of the space, of the curve, and of the weight. As an example, we consider weighted Nakano spaces $L_{w}^{p(\cdot)}$ (weighted Lebesgue spaces with variable exponent). Moreover, our necessary conditions become also sufficient for weighted Nakano spaces over nice curves whenever $w$ is a Khvedelidze weight, and the variable exponent $p(t)$ satisfies the estimate $|p(\tau)-p(t)| \leq A /(-\log |\tau-t|)$.
\end{abstract}

\section{INTRODUCTION}

Let $\Gamma$ be a Jordan curve, that is, a curve that homeomorphic to a circle. We suppose that $\Gamma$ is rectifiable. We equip $\Gamma$ with Lebesgue length measure $|d \tau|$ and the counter-clockwise orientation. The Cauchy singular integral of a measurable function $f: \Gamma \rightarrow \mathbb{C}$ is defined by

$$
(S f)(t):=\lim _{R \rightarrow 0} \frac{1}{\pi i} \int_{\Gamma \backslash \Gamma(t, R)} \frac{f(\tau)}{\tau-t} d \tau \quad(t \in \Gamma),
$$

where the "portion" $\Gamma(t, R)$ is

$$
\Gamma(t, R):=\{\tau \in \Gamma:|\tau-t|<R\} \quad(R>0) .
$$

It is well known that $(S f)(t)$ exists a.e. on $\Gamma$ whenever $f$ is integrable (see 11 , Theorem 2.22]). A measurable function $w: \Gamma \rightarrow[0, \infty]$ is referred to as a weight if $0<w(t)<\infty$ a.e. on $\Gamma$. The Cauchy singular integral generates a bounded linear operator $S$ on the weighted Lebesgue space $L_{w}^{p}(1<p<\infty)$ with the norm

$$
\|f\|_{L_{w}^{p}}:=\left(\int_{\Gamma}|f(\tau)|^{p} w^{p}(\tau)|d \tau|\right)^{1 / p}
$$

if and only if $w$ is a Muckenhoupt weight $\left(w \in A_{p}(\Gamma)\right)$, that is,

$$
\sup _{t \in \Gamma} \sup _{R>0}\left(\frac{1}{R} \int_{\Gamma(t, R)} w^{p}(\tau)|d \tau|\right)^{1 / p}\left(\frac{1}{R} \int_{\Gamma(t, R)} w^{-p^{\prime}}(\tau)|d \tau|\right)^{1 / p^{\prime}}<\infty, \frac{1}{p}+\frac{1}{p^{\prime}}=1
$$

Date: February 18, 2003.

2000 Mathematics Subject Classification. Primary 45E05, 46E30; Secondary 47B35, 47A53, $47 \mathrm{~A} 68$.

Key words and phrases. Weighted Banach function space, Nakano space, singular integral operator, Fredholmness, Carleson curve, indices of submultiplicative function.

The author is partially supported by F.C.T. (Portugal) grants POCTI 34222/MAT/2000 and PRAXIS XXI/BPD/22006/99. 
(see, e.g., 3. Theorem 4.15]). By Hölder's inequality, if $w \in A_{p}(\Gamma)$, then $\Gamma$ is a Carleson (or Ahlfors-David regular) curve, that is,

$$
C_{\Gamma}:=\sup _{t \in \Gamma} \sup _{R>0} \frac{|\Gamma(t, R)|}{R}<\infty,
$$

where $|\Omega|$ denotes the measure of a measurable set $\Omega \subset \Gamma$. The constant $C_{\Gamma}$ is said to be the Carleson constant. We denote by $P C$ the Banach algebra of all piecewise continuous functions on the curve $\Gamma$ : by definition, $a$ is in $P C$ if and only if $a$ is in $L^{\infty}$ and the one-sided limits

$$
a(t \pm 0):=\lim _{\tau \rightarrow t \pm 0} a(\tau)
$$

exist for every $t \in \Gamma$.

A bounded linear operator $A$ on a Banach space is said to be semi-Fredholm if its image is closed and at least one of the so-called defect numbers

$$
n(A):=\operatorname{dim} \operatorname{ker} A, \quad d(A):=\operatorname{dim} \operatorname{ker} A^{*}
$$

is finite. A semi-Fredholm operator $A$ is called Fredholm if both $n(A)$ and $d(A)$ are finite. In this case the difference $n(A)-d(A)$ is referred to as the index of the operator $A$. Basic properties of (semi)-Fredholm operators are discussed in [5] 16. 40, and in many other monographs. form

The study of Fredholmness of one-dimensional singular integral operators of the

$$
R_{a}:=a P_{+}+P_{-}, \quad a \in P C, \quad P_{ \pm}:=(I \pm S) / 2
$$

on Lebesgue spaces with power (Khvedelidze) weights

$$
\varrho(t):=\prod_{k=1}^{n}\left|t-\tau_{k}\right|^{\lambda_{k}}, \quad \tau_{k} \in \Gamma, \quad k \in\{1, \ldots, n\}, \quad n \in \mathbb{N},
$$

over Lyapunov curves started in the fiftieth with B. V. Khvedelidze 27] and was continued in the sixties by H. Widom, I. B. Simonenko, I. Gohberg and N. Krupnik, and others. The history and corresponding references can be found, e.g., in 3, 16, [21, 28, 40. In the beginning of nineties, I. Spitkovsky proved Fredholm criteria for singular integral operators with piecewise continuous coefficients on Lebesgue spaces with Muckenhoupt weights over smooth curves [52]. In the middle of nineties, A. Böttcher and Yu. I. Karlovich accomplished the Fredholm theory for the algebra of singular integral operators with piecewise continuous coefficients on Lebesgue spaces with Muckenhoupt weights over general Carleson curves. These results are documented in [3]; see also the brief but nice presentation in [4].

Lebesgue spaces $L^{p}, 1 \leq p \leq \infty$, are the simplest examples of so-called Banach function spaces introduced by W. A. J. Luxemburg in 1955. This scale of spaces includes Orlicz, Lorentz, and all other rearrangement-invariant spaces. By analogy with weighted Lebesgue spaces, for a Banach function space $X$ and a weight $w$, it is possible to define the weighted Banach function space

$$
X_{w}:=\{f \text { is measurable on } \Gamma \text { and } f w \in X\} .
$$

Under some restrictions on the weight $w$, the space $X_{w}$ is itself a Banach function space, although if $X$ is a rearrangement-invariant Banach function space, then $X_{w}$ is not necessarily rearrangement-invariant (even if $X$ is a Lebesgue space). 
Another interesting class of Banach function spaces which are not rearrangementinvariant constituted by Nakano spaces $L^{p(\cdot)}$ (generalized Lebesgue spaces with variable exponent). For details and references, see Section 2

Unfortunately, few is known about the boundedness of $S$ on general weighted Banach function spaces $X_{w}$. As far as we know, even a criterion for the boundedness of $S$ on Orlicz spaces $L_{w}^{\varphi}$ with general weights $w$ over general Carleson curves is unknown at the moment (February of 2003). We proved necessary conditions for the boundedness of $S$ on weighted rearrangement-invariant Banach function spaces [24. Theorem 3.2] in terms of an analog of the Muckenhoupt class. On the other hand, if a weight $w$ belongs to the Muckenhoupt classes $A_{1 / \alpha_{X}}(\Gamma)$ and $A_{1 / \beta_{X}}(\Gamma)$ where $\alpha_{X}, \beta_{X} \in(0,1)$ are the Boyd indices of a rearrangement-invariant Banach function space $X$, then $S$ is bounded on the weighted rearrangement-invariant Banach function space $X_{w}$ (see [26, Theorem 4.5]).

On the basis of these boundedness results, following the approach of A. Böttcher, Yu. Karlovich, and I. Spitkovsky, the author proved separately necessary and sufficient conditions for Fredholmness of singular integral operators with piecewise continuous coefficients on weighted rearrangement-invariant Banach function spaces 25. 26. Under some restrictions on spaces, curves, and weights, these conditions coincide, that is, become criteria. In that cases, the Banach algebra of singular integral operators with piecewise continuous coefficients is also studied [26].

Very recently V. M. Kokilashvili and S. G. Samko have proved criteria for the boundedness of $S$ on Nakano spaces $L_{\varrho}^{p(\cdot)}$ with Khvedelidze weights $\varrho$ over Lyapunov curves or Radon curves without cusps provided the variable exponent $p$ satisfies the estimate

$$
|p(\tau)-p(t)| \leq A /(-\log |\tau-t|), \quad \tau, t \in \Gamma, \quad|\tau-t| \leq 1 / 2
$$

(see 30, Theorem 2] or Theorem 6.2). With the help of this key result, they have proved Fredholm criteria for the operator $a P_{+}+b P_{-}$with piecewise continuous functions $a, b$ having finite numbers of jumps on (non-weighted) Nakano spaces $L^{p(\cdot)}$ (see [31, Theorem A]).

For an arbitrary weight $w$ and an arbitrary Banach function space $X$, we define the weighted Banach function space $X_{w}$. Assume that

(B) the Cauchy singular integral operator $S$ is bounded on $X_{w}$;

(R) $X_{w}$ is reflexive.

We show that property (B) implies the condition $A_{X}(\Gamma)$ of Muckenhoupt type. In that case $X_{w}$ is itself a Banach function space. Under the assumptions (B) and $(\mathrm{R})$ we prove necessary conditions for Fredholmness of singular integral operators $R_{a}$ with piecewise continuous coefficients $a$ in the weighted Banach function spaces $X_{w}$. This result generalizes corresponding necessary conditions in 25, Theorem 4.2]. As an example, we consider these necessary conditions in Nakano spaces $L_{w}^{p(\cdot)}$ with general weights $w$. They have almost the same form as in the case of Lebesgue spaces $L_{w}^{p}$ with Muckenhoupt weights over Carleson curves (see [3, Proposition 7.3]). We should only replace the constant $p$ (for weighted Lebesgue spaces $L_{w}^{p}$ ) by the value $p(t)$ of the variable exponent $p(\cdot)$ at each point $t \in \Gamma$ (for weighted Nakano spaces $L_{w}^{p(\cdot)}$ ). Our approach is based on a local principle of Simonenko type, the Wiener-Hopf factorization of local representatives, and the theory of submultiplicative functions associated with local properties of the curve, of the weight, and of the space. Using of the local principle allows us to consider 
coefficients $a$ having a countable number of jumps (in contrast to [31, where only a finite number of jumps is allowed).

The paper is organized as follows. In Section 2 we collect necessary preliminaries on weighted Banach function spaces $X_{w}$ and Nakano spaces $L^{p(\cdot)}$. In Section 3 we define an analog of the Muckenhoupt class $A_{p}(\Gamma)$, replacing the norm in $L^{p}$ by the norm in a Banach function space $X$. We denote this class by $A_{X}(\Gamma)$. We show that if $w \in A_{X}(\Gamma)$ and $1 \in A_{X}(\Gamma)$, then $\log w$ has bounded mean oscillation. In Section 4 we remind the definitions and some properties of submultiplicative functions associated with the local behavior of the curve, of the weight, and of the space. In Section 5 we study inequalities between the indices of submultiplicative functions defined in Section 4 We investigate so-called indicator functions $\alpha_{t}^{*}, \beta_{t}^{*}$ and $\alpha_{t}, \beta_{t}$ of the triple $(\Gamma, X, w)$ and of the pair $(\Gamma, w)$, respectively. In particular, we show that if $X$ is a Nakano space $L^{p(\cdot)}$ with a variable exponent $p(\cdot)$ satisfying (1.3), then we can separate the influence of the space from the influence of the weight and the curve, that is, $\alpha_{t}^{*}(x)=1 / p(t)+\alpha_{t}(x), \beta_{t}^{*}(x)=1 / p(t)+\beta_{t}(x)$ for $x \in \mathbb{R}$ such that $\left|(\tau-t)^{y+i x}\right| w(\tau) \in A_{L^{p(\cdot)}}(\Gamma, t)$, where $A_{L^{p(\cdot)}}(\Gamma, t)$ is the local analog of $A_{L^{p(\cdot)}}(\Gamma)$. So, weighted Nakano spaces satisfy the "disintegration condition" in the terminology of [24, 26].

In Section 6 we prove that the condition $w \in A_{X}(\Gamma)$ is necessary for the boundedness of the Cauchy singular integral operator $S$ on the weighted Banach function spaces $X_{w}$. Further we extend basic results on the Fredholmness of singular integral operators with bounded measurable coefficients (the local principle, the theorem about a Wiener-Hopf factorization, etc.) to weighted Banach function spaces satisfying Axioms (B) and (R). These results are natural extensions of the classical theory for Lebesgue spaces with Khvedelidze weights over Lyapunov curves (see, e.g., [16, Ch. 7-8] or 40, Ch. 4]). A canonical local representative $g_{t, \gamma}(t \in \Gamma, \gamma \in \mathbb{C})$ for a piecewise continuous function is constructed in Section 7 We prove separately necessary and sufficient conditions for factorability of $g_{t, \gamma}$ in the weighted Banach function space $X_{w}$. On the basis of our necessary conditions for factorability, with the help of the results of Section [6] we prove necessary conditions for Fredholmness of the singular integral operator $R_{a}=a P_{+}+P_{-}$with $a \in P C$ in $X_{w}$. These conditions are formulated in terms of the indicator functions $\alpha_{t}^{*}$ and $\beta_{t}^{*}$ defined in Section 15 In Section 8 we reformulate these necessary conditions for weighted Nakano spaces $L_{w}^{p(\cdot)}$ with general weights $w$ and variable exponents satisfying (1.3) in terms of simpler indicator functions $\alpha_{t}$ and $\beta_{t}$. With the help of the boundedness criteria by V. M. Kokilashvili and S. G. Samko [30, Theorem 2], we prove that the latter necessary conditions become also sufficient if $w=\varrho$ is a Khvedelidze weight and $\Gamma$ is either a Lyapunov Jordan curve or a Radon Jordan curve without cusps.

\section{Weighted BANACH FUnCTION SPACES}

2.1. Banach function spaces. Let $\Gamma$ be a rectifiable Jordan (i.e., homeomorphic to a circle) curve equipped with Lebesgue length measure $|d \tau|$. The set of all measurable complex-valued functions on $\Gamma$ is denoted by $\mathcal{M}$. Let $\mathcal{M}^{+}$be the subset of functions in $\mathcal{M}$ whose values lie in $[0, \infty]$. The characteristic function of a measurable set $E \subset \Gamma$ is denoted by $\chi_{E}$.

Definition 2.1. (W. A. J. Luxemburg, 1955, see [1. Ch. 1, Definition 1.1]). A mapping $\rho: \mathcal{M}^{+} \rightarrow[0, \infty]$ is called a Banach function norm if, for all functions $f, g, f_{n}(n \in \mathbb{N})$ in $\mathcal{M}^{+}$, for all constants $a \geq 0$, and for all measurable subsets $E$ 
of $\Gamma$, the following properties hold:

$$
\begin{aligned}
& \rho(f)=0 \Leftrightarrow f=0 \text { a.e., } \quad \rho(a f)=a \rho(f), \quad \rho(f+g) \leq \rho(f)+\rho(g), \\
& 0 \leq g \leq f \text { a.e. } \Rightarrow \rho(g) \leq \rho(f) \quad \text { (the lattice property), } \\
& 0 \leq f_{n} \uparrow f \text { a.e. } \Rightarrow \rho\left(f_{n}\right) \uparrow \rho(f) \quad \text { (the Fatou property), } \\
& \rho\left(\chi_{E}\right)<\infty, \\
& \int_{E} f(\tau)|d \tau| \leq C_{E} \rho(f)
\end{aligned}
$$

with $C_{E} \in(0, \infty)$ may depend on $E$ and $\rho$ but is independent of $f$.

When functions differing only on a set of measure zero are identified, the set $X$ of all functions $f \in \mathcal{M}$ for which $\rho(|f|)<\infty$ is called a Banach function space. For each $f \in X$, the norm of $f$ is defined by

$$
\|f\|_{X}:=\rho(|f|) .
$$

The set $X$ under the natural linear space operations and under this norm becomes a Banach space (see [1, Ch. 1, Theorems 1.4 and 1.6]).

If $\rho$ is a Banach function norm, its associate norm $\rho^{\prime}$ is defined on $\mathcal{M}^{+}$by

$$
\rho^{\prime}(g):=\sup \left\{\int_{\Gamma} f(\tau) g(\tau)|d \tau|: f \in \mathcal{M}^{+}, \rho(f) \leq 1\right\}, \quad g \in \mathcal{M}^{+} .
$$

It is a Banach function norm itself [1, Ch. 1, Theorem 2.2]. The Banach function space $X^{\prime}$ determined by the Banach function norm $\rho^{\prime}$ is called the associate space (Köthe dual) of $X$. The associate space $X^{\prime}$ is a subspace of the dual space $X^{*}$. The construction of the associate space implies the following Hölder inequality for Banach function spaces.

Lemma 2.2. (see 11, Ch. 1, Theorem 2.4]). Let $X$ be a Banach function space and $X^{\prime}$ be its associate space. If $f \in X$ and $g \in X^{\prime}$, then $f g$ is integrable and

$$
\|f g\|_{L^{1}} \leq\|f\|_{X}\|g\|_{X^{\prime}}
$$

2.2. Rearrangement-invariant Banach function spaces. Let $\mathcal{M}_{0}$ and $\mathcal{M}_{0}^{+}$ be the classes of a.e. finite functions in $\mathcal{M}$ and $\mathcal{M}^{+}$, respectively. Two functions $f, g \in \mathcal{M}_{0}$ are said to be equimeasurable if

$$
|\{\tau \in \Gamma:|f(\tau)|>\lambda\}|=|\{\tau \in \Gamma:|g(\tau)|>\lambda\}| \text { for all } \quad \lambda \geq 0 .
$$

A Banach function norm $\rho: \mathcal{M}^{+} \rightarrow[0, \infty]$ is called rearrangement-invariant if for every pair of equimeasurable functions $f, g \in \mathcal{M}_{0}^{+}$the equality $\rho(f)=\rho(g)$ holds. In that case, the Banach function space $X$ generated by $\rho$ is said to be a rearrangement-invariant Banach function space (or simply rearrangement-invariant space). Lebesgue, Orlicz, Lorentz, and Lorentz-Orlicz spaces are classical examples of rearrangement-invariant Banach function spaces (see, e.g., [1] and the references therein).

If $X$ is an arbitrary rearrangement-invariant Banach function space and $X^{\prime}$ is its associate space, then for a measurable set $E \subset \Gamma$,

$$
\left\|\chi_{E}\right\|_{X}\left\|\chi_{E}\right\|_{X^{\prime}}=|E|
$$

(see, e.g., 11 Ch. 2, Theorem 5.2]). 
2.3. Nakano spaces $L^{p(\cdot)}$. Function spaces $L^{p(\cdot)}$ of Lebesgue type with variable exponent $p$ were studied for the first time probably by W. Orlicz [45] in 1931. Inspired by the successful theory of Orlicz spaces, H. Nakano defined in the late forties 43, 44, so-called modular spaces. He considered the space $L^{p(\cdot)}$ as an example of modular spaces. J. Musielak and W. Orlicz 42 in 1959 extended the definition of modular spaces by H. Nakano. Actually, that paper was the starting point for the theory of Musielak-Orlicz spaces (generalized Orlicz spaces generated by Young functions with a parameter), see 41].

Let $p: \Gamma \rightarrow[1, \infty)$ be a measurable function. Consider the convex modular (see [41, Ch. 1] for definitions and properties)

$$
m(f, p):=\int_{\Gamma}|f(\tau)|^{p(\tau)}|d \tau| .
$$

Denote by $L^{p(\cdot)}$ the set of all measurable complex-valued functions $f$ on $\Gamma$ such that $m(\lambda f, p)<\infty$ for some $\lambda=\lambda(f)>0$. This set becomes a Banach space with respect to the Luxemburg-Nakano norm

$$
\|f\|_{L^{p(\cdot)}}:=\inf \{\lambda>0: m(f / \lambda, p) \leq 1\}
$$

(see, e.g., 41, Ch. 2]). So, the spaces $L^{p(\cdot)}$ are a special case of Musielak-Orlicz spaces. Sometimes the spaces $L^{p(\cdot)}$ are referred to as Nakano spaces (see, e.g., 13, p. 151], 19 p. 179]). We will follow this tradition. Clearly, if $p(\cdot)=p$ is constant, then the Nakano space $L^{p(\cdot)}$ is isometrically isomorphic to the Lebesgue space $L^{p}$. Therefore, sometimes $L^{p(\cdot)}$ are called generalized Lebesgue spaces with variable exponent.

Lemma 2.3. (see, e.g., 12, Proposition 1.3]). Let $p: \Gamma \rightarrow[1, \infty)$ be a measurable function. The Nakano space $L^{p(\cdot)}$ is a Banach function space.

It is not difficult to show that $L^{p(\cdot)}$ is not rearrangement-invariant, in general.

The following result on the reflexivity and duality of Nakano spaces was precisely stated in [32 Theorem 2.3 and Corollary 2.7], although it can be obtained from more general results for Musielak-Orlicz spaces [41, Ch. 1-2] (see also [45).

Lemma 2.4. Let $p: \Gamma \rightarrow[1, \infty)$ be a measurable function. If

$$
1<\underset{t \in \Gamma}{\operatorname{essinf}} p(t) \leq \operatorname{ess}_{t \in \Gamma} \sup p(t)<\infty,
$$

then the Nakano space $L^{p(\cdot)}$ is reflexive. Its associate space coincides (up to the equivalence of the norms) with the Nakano space $L^{p^{\prime}(\cdot)}$, where

$$
p^{\prime}(\tau):=\frac{p(\tau)}{p(\tau)-1} .
$$

Finally, Nakano spaces are important in applications to fluid dynamics [48].

2.4. Weighted Banach function spaces. Let $X$ be a Banach function space generated by a Banach function norm $\rho$ and let $w: \Gamma \rightarrow[0, \infty]$ be a weight. Define the mapping $\rho_{w}: \mathcal{M}^{+} \rightarrow[0, \infty]$ and the set $X_{w}$ by

$$
\rho_{w}(f):=\rho(f w) \quad\left(f \in \mathcal{M}^{+}\right), \quad X_{w}:=\left\{f \in \mathcal{M}^{+}: \quad f w \in X\right\} .
$$


Lemma 2.5. (a) $\rho_{w}$ satisfies Axioms (A1)-(A3) in Definition 2.1 and $X_{w}$ is a linear normed space with respect to the norm

$$
\|f\|_{X_{w}}:=\rho_{w}(|f|)=\rho(|f w|)=\|f w\|_{X}
$$

(b) if $w \in X$ and $1 / w \in X^{\prime}$, then $\rho_{w}$ is a Banach function norm and $X_{w}$ is a Banach function space generated by $\rho_{w}$. Moreover,

$$
L^{\infty} \subset X_{w} \subset L^{1}
$$

(c) if $w \in X$ and $1 / w \in X^{\prime}$, then $X_{1 / w}^{\prime}$ is the associate space for the Banach function space $X_{w}$.

Proof. Part (a) follows from Axioms (A1)-(A3) for the Banach function norm $\rho$ and the fact that $0<w(\tau)<\infty$ a.e. on $\Gamma$.

(b) If $w \in X$, then by Axiom (A2) for $\rho$, we get $w \chi_{E} \in X$ for every measurable set $E$ of $\Gamma$. Therefore, $\rho_{w}\left(\chi_{E}\right)=\rho\left(w \chi_{E}\right)<\infty$. Thus, $\rho_{w}$ satisfies Axiom (A4). By Hölder's inequality (see Lemma 2.2) and Axiom (A2) for $\rho$, we have

$$
\begin{aligned}
\int_{E} f(\tau)|d \tau| & =\int_{\Gamma}\left(f(\tau) w(\tau) \chi_{E}(\tau)\right) \frac{\chi_{E}(\tau)}{w(\tau)}|d \tau| \\
& \leq \rho\left(f w \chi_{E}\right) \rho^{\prime}\left(\chi_{E} / w\right) \leq \rho(f w) \rho^{\prime}\left(\chi_{E} / w\right)=: C_{E} \rho_{w}(f),
\end{aligned}
$$

where $C_{E}:=\rho^{\prime}\left(\chi_{E} / w\right) \in(0, \infty)$. This constant, clearly, depends on $\rho, w$ (and thus on $\rho_{w}$ ) and $E$, but it is independent of $f$. Therefore, $\rho_{w}$ satisfies Axiom (A5). Thus, $\rho_{w}$ is a Banach function norm and $X_{w}$ is a Banach function space.

From (2.2) and Axiom (A2) for $X^{\prime}$ it follows that

$$
\|f\|_{L^{1}} \leq\|f\|_{X_{w}}\|1 / w\|_{X^{\prime}}, \quad f \in X_{w} .
$$

Hence, $X_{w} \subset L^{1}$, in view of $1 / w \in X^{\prime}$. On the other hand, for $f \in L^{\infty}$,

$$
0 \leq|f(\tau)| \leq\|f\|_{\infty} \quad \text { a.e. on } \Gamma \text {. }
$$

By Axioms (A2) and (A1) for $\rho_{w}$, we have

$$
\|f\|_{X_{w}}=\rho_{w}(|f|) \leq \rho_{w}\left(\|f\|_{\infty}\right)=\|f\|_{\infty} \rho_{w}(1)=\|f\|_{\infty}\|w\|_{X} .
$$

Thus, $L^{\infty} \subset X_{w}$, in view of $w \in X$. Part (b) is proved.

(c) For $g \in \mathcal{M}^{+}$, we have

$$
\begin{aligned}
\left(\rho_{w}\right)^{\prime}(g) & =\sup \left\{\int_{\Gamma} f(\tau) g(\tau)|d \tau|: \quad f \in \mathcal{M}^{+}, \quad \rho_{w}(f) \leq 1\right\} \\
& =\sup \left\{\int_{\Gamma}(f(\tau) w(\tau))\left(\frac{g(\tau)}{w(\tau)}\right)|d \tau|: \quad f \in \mathcal{M}^{+}, \quad \rho(f w) \leq 1\right\} \\
& =\sup \left\{\int_{\Gamma} h(\tau)\left(\frac{g(\tau)}{w(\tau)}\right)|d \tau|: \quad h \in \mathcal{M}^{+}, \quad \rho(h) \leq 1\right\} \\
& =\rho^{\prime}(g / w) .
\end{aligned}
$$

Hence, $\left(X_{w}\right)^{\prime}=X_{1 / w}^{\prime}$.

We will refer to the normed space $X_{w}$ as a weighted Banach function space generated by the Banach function space $X$ and the weight $w$. From Lemma 2.5(b) it follows that the weighted Banach function space $X_{w}$ is a Banach function space itself whenever $w \in X$ and $1 / w \in X^{\prime}$.

For other definition (different from our) of weighted Banach function spaces, see, e.g., 34, 37]. 
2.5. Separability and reflexivity of weighted Banach function spaces. A function $f$ in a Banach function space $X$ is said to have absolutely continuous norm in $X$ if $\left\|f \chi_{E_{n}}\right\|_{X} \rightarrow 0$ for every sequence $\left\{E_{n}\right\}_{n=1}^{\infty}$ of measurable sets on $\Gamma$ satisfying $\chi_{E_{n}} \rightarrow 0$ a.e. on $\Gamma$ as $n \rightarrow \infty$. If all functions $f \in X$ have this property, then the space $X$ itself is said to have absolutely continuous norm (see [1. Ch. 1, Section 3]).

In this subsection we assume that $X$ is a Banach function space and $w$ is a weight such that $w \in X$ and $1 / w \in X^{\prime}$. Then, by Lemma 2.5(b), the weighted Banach function space $X_{w}$ is itself a Banach function space.

Proposition 2.6. If $X$ has absolutely continuous norm, then $X_{w}$ has absolutely continuous norm too.

Proof. If $f \in X_{w}$, then $f w \in X$ has absolutely continuous norm in $X$. Therefore, $\left\|f \chi_{E_{n}}\right\|_{X_{w}}=\left\|f w \chi_{E_{n}}\right\|_{X} \rightarrow 0$ for every sequence $\left\{E_{n}\right\}_{n=1}^{\infty}$ of measurable sets on $\Gamma$ satisfying $\chi_{E_{n}} \rightarrow 0$ a.e. on $\Gamma$ as $n \rightarrow \infty$. Thus, $f \in X_{w}$ has absolutely continuous norm in $X_{w}$.

From Lemma 2.5 and [1, Ch. 1, Corollaries 4.3, 4.4] we obtain the following.

Lemma 2.7. (a) The Banach space dual $\left(X_{w}\right)^{*}$ of the weighted Banach function space $X_{w}$ is isometrically isomorphic to the associate space $X_{1 / w}^{\prime}$ if and only if $X_{w}$ has absolutely continuous norm. If $X_{w}$ has absolutely continuous norm, then the general form of a linear functional on $X_{w}$ is given by

$$
G(f):=\int_{\Gamma} f(\tau) \overline{g(\tau)}|d \tau|, \quad g \in X_{1 / w}^{\prime}, \quad \text { and } \quad\|G\|_{\left(X_{w}\right)^{*}}=\|g\|_{X_{1 / w}^{\prime}} .
$$

(b) The weighted Banach function space $X_{w}$ is reflexive if and only if both $X_{w}$ and $X_{1 / w}^{\prime}$ have absolutely continuous norm.

Corollary 2.8. If $X$ is reflexive, then $X_{w}$ is reflexive.

Proof. If $X$ is reflexive, then, by [1, Ch. 1, Corollary 4.4], both $X$ and $X^{\prime}$ have absolutely continuous norm. In that case, due to Proposition [2.6 both $X_{w}$ and $X_{1 / w}^{\prime}$ have absolutely continuous norm. By Lemma 2.7(b), $X_{w}$ is reflexive.

Since Lebesgue length measure $|d \tau|$ is separable (for the definition of a separable measure, see, e.g., 11, p. 27] or [20, Section 6.10]), from Lemma 2.5] and [1, Ch. 1, Corollary 5.6] we immediately get the following criterion.

Lemma 2.9. The weighted Banach function space $X_{w}$ is separable if and only if it has absolutely continuous norm.

We denote by $C$ the set of all continuous functions on $\Gamma$ and by $\mathcal{R}$ the set of all rational functions without poles on the curve $\Gamma$. With the help of Lemmas 2.7 and 2.9 literally repeating the proof of [25. Lemma 1.3], one can get the following.

Lemma 2.10. The weighted Banach function space $X_{w}$ is separable if and only if $C$ is dense in $X_{w}$.

Corollary 2.11. If $X_{w}$ (or $X$ ) is reflexive, then $\mathcal{R}$ is dense in $X_{w}$ and in its associate space $X_{1 / w}^{\prime}$.

Proof. If $X_{w}$ is reflexive, then by Lemmas 2.7 (b) and 2.9 both $X_{w}$ and $X_{1 / w}^{\prime}$ are separable. This implies that $C$ is dense in $X_{w}$ and in $X_{1 / w}^{\prime}$, due to Lemma 2.10 In view of the Mergelyan theorem (see, e.g., 14 Ch. III, Section 2]), every function 
in $C$ may uniformly be approximated by functions in $\mathcal{R}$. Thus, $\mathcal{R}$ is dense in $X_{w}$ and in $X_{1 / w}^{\prime}$. If $X$ is reflexive, we need first apply Corollary 2.8 and then repeat the above arguments.

\section{Analogs of the Muckenhoupt Class}

3.1. Definitions. Let $X$ be a Banach function space. Fix $t \in \Gamma$. For a weight $w: \Gamma \rightarrow[0, \infty]$, put

$$
B_{t, R}(w):=\frac{1}{R}\left\|w \chi_{\Gamma(t, R)}\right\|_{X}\left\|\chi_{\Gamma(t, R)} / w\right\|_{X^{\prime}} \quad(R>0),
$$

where $\chi_{\Gamma(t, R)}$ is the characteristic function of the portion $\Gamma(t, R)$. Consider the following classes of weights:

$$
A_{X}(\Gamma, t):=\left\{w: \sup _{R>0} B_{t, R}(w)<\infty\right\}, \quad A_{X}(\Gamma):=\left\{w: \sup _{t \in \Gamma} \sup _{R>0} B_{t, R}(w)<\infty\right\} .
$$

Obviously, $A_{X}(\Gamma) \subset A_{X}(\Gamma, t)$ for $t \in \Gamma$. If $X$ is a Lebesgue space $L^{p}, p \in(1, \infty)$, then $A_{X}(\Gamma)$ is the Muckenhoupt class $A_{p}(\Gamma)$. For a detailed discussion of Muckenhoupt weights on curves, see, e.g., $\left[3\right.$. The classes $A_{X}(\Gamma, t)$ and $A_{X}(\Gamma)$ were defined in 24] (see also 22, 25]) for rearrangement-invariant spaces $X$. Here we assume only that $X$ is a Banach function space. Ours definition is similar to a definition in 2]. For others generalizations (different from our) of the Muckenhoupt class $A_{p}(\Gamma)$ in the setting of Orlicz and Lorentz spaces, see, e.g., [15, 29] and in the setting of Banach function spaces, see 34 .

With the help of Hölder's inequality (see Lemma 2.2), it is easy to show that $w \in A_{X}(\Gamma, t)$ implies

$$
C_{\Gamma, t}:=\sup _{R>0} \frac{|\Gamma(t, R)|}{R}<\infty .
$$

We say that a rectifiable Jordan curve $\Gamma$ is locally a Carleson curve at the point $t \in \Gamma$ if (3.1) is satisfied. In that case the constant $C_{\Gamma, t}$ is referred to as the local Carleson constant at the point $t \in \Gamma$. Analogously, if $w \in A_{X}(\Gamma)$, then

$$
C_{\Gamma}=\sup _{t \in \Gamma} C_{\Gamma, t}<\infty
$$

that is, $\Gamma$ is a Carleson curve.

3.2. Bounded and vanishing mean oscillation. Let $\Gamma$ be a rectifiable Jordan curve. Let $f: \Gamma \rightarrow[-\infty, \infty]$ and $f \in L^{1}(\Gamma)$. Suppose $t \in \Gamma, \delta \in(0, \infty]$, and $R \in(0, \infty)$. Put

$$
\begin{aligned}
\Omega_{t}(f, R) & :=\frac{1}{|\Gamma(t, R)|} \int_{\Gamma(t, R)} f(\tau)|d \tau| \\
M_{\delta, t}(f) & :=\sup _{0<R<\delta} \frac{1}{|\Gamma(t, R)|} \int_{\Gamma(t, R)}\left|f(\tau)-\Omega_{t}(f, R)\right||d \tau| .
\end{aligned}
$$

A function $f$ is said to be of bounded mean oscillation at the point $t \in \Gamma$ if $\|f\|_{*, t}:=M_{\infty, t}(f)<\infty$. In this case we will write $f \in B M O(\Gamma, t)$. A function $f \in B M O(\Gamma, t)$ has vanishing mean oscillation at the point $t \in \Gamma$ if

$$
\lim _{\delta \rightarrow 0} M_{\delta, t}(f)=0 .
$$

In that case we will write $f \in V M O(\Gamma, t)$. 
One says that a function $f: \Gamma \rightarrow[-\infty, \infty]$ is of bounded mean oscillation on $\Gamma$ if $f \in B M O(\Gamma, t)$ for all $t \in \Gamma$ and

$$
\|f\|_{*}:=\sup _{t \in \Gamma}\|f\|_{*, t}<\infty
$$

The class of functions of bounded mean oscillation on $\Gamma$ is denoted by $B M O(\Gamma)$. A function $f \in B M O(\Gamma)$ is said to be of vanishing mean oscillation on $\Gamma$ if

$$
\lim _{\delta \rightarrow 0} \sup _{t \in \Gamma} M_{\delta, t}(f)=0 .
$$

The class of functions of vanishing mean oscillation on $\Gamma$ is denoted by $V M O(\Gamma)$. Clearly, $B M O(\Gamma) \subset B M O(\Gamma, t)$ and $V M O(\Gamma) \subset V M O(\Gamma, t)$ for every $t \in \Gamma$.

\subsection{Bounded mean oscillation of logarithms of weights. Let}

$$
d_{t}:=\max _{\tau \in \Gamma}|\tau-t| \text {. }
$$

For a weight $w: \Gamma \rightarrow[0, \infty]$ such that $w \in X(\Gamma)$ and $1 / w \in X^{\prime}(\Gamma)$, we have $w, 1 / w \in L^{1}(\Gamma)$. Then, taking into account the obvious inequality $|\log x| \leq x+1 / x$ for $x \in(0, \infty)$, we deduce that $\log w \in L^{1}$. For $t \in \Gamma$ and $R>0$, put

$$
\begin{aligned}
C(w, t, R) & :=\exp \left(-\Omega_{t}(\log w, R)\right) \frac{\left\|w \chi_{\Gamma(t, R)}\right\|_{X}\left\|\chi_{\Gamma(t, R)}\right\|_{X^{\prime}}}{|\Gamma(t, R)|}, \\
C^{\prime}(w, t, R) & :=\exp \left(\Omega_{t}(\log w, R)\right) \frac{\left\|\chi_{\Gamma(t, R)}\right\|\left\|_{X}\right\| \chi_{\Gamma(t, R)} / w \|_{X^{\prime}}}{|\Gamma(t, R)|} .
\end{aligned}
$$

Clearly, these quantities are well defined.

Lemma 3.1. (a) If $w \in A_{X}(\Gamma, t)$ and $1 \in A_{X}(\Gamma, t)$, then

$$
1 \leq \sup _{R>0} C(w, t, R)<\infty, \quad 1 \leq \sup _{R>0} C^{\prime}(w, t, R)<\infty .
$$

(b) If $w \in A_{X}(\Gamma)$ and $1 \in A_{X}(\Gamma)$, then

$$
1 \leq \sup _{t \in \Gamma} \sup _{R>0} C(w, t, R)<\infty, \quad 1 \leq \sup _{t \in \Gamma} \sup _{R>0} C^{\prime}(w, t, R)<\infty .
$$

Proof. The proof is developed by similarity to 25. Lemma 1.5]. Applying Jensen's inequality (see, e.g., [33, p. 78]) and Hölder's inequality (see Lemma [2.2), we obtain

$$
\exp \left(\Omega_{t}(\log w, R)\right) \leq \frac{1}{|\Gamma(t, R)|} \int_{\Gamma(t, R)} w(\tau)|d \tau| \leq \frac{\left\|w \chi_{\Gamma(t, R)}\right\|_{X}\left\|\chi_{\Gamma(t, R)}\right\|_{X^{\prime}}}{|\Gamma(t, R)|}
$$

Hence,

$$
1 \leq C(w, t, R), \quad R>0 .
$$

Analogously,

$$
1 \leq C^{\prime}(w, t, R), \quad R>0 .
$$

Inequalities (3.4) and 3.5 imply that 3.2) is equivalent to

$$
\sup _{R>0}\left(C(w, t, R) C^{\prime}(w, t, R)\right)<\infty
$$

and (3.3) is equivalent to

$$
\sup _{t \in \Gamma} \sup _{R>0}\left(C(w, t, R) C^{\prime}(w, t, R)\right)<\infty .
$$


Since $\Gamma(t, R)=\Gamma$ for $R>d_{t}$, we have for every $t \in \Gamma$,

$$
\begin{gathered}
\sup _{R>0} B_{t, R}(w)=\sup _{R \in\left(0,2 d_{t}\right]} B_{t, R}(w), \quad \sup _{R>0} B_{t, R}(1)=\sup _{R \in\left(0,2 d_{t}\right]} B_{t, R}(1), \\
\sup _{R>0}\left(C(w, t, R) C^{\prime}(w, t, R)\right)=\sup _{0<R \leq 2 d_{t}}\left(C(w, t, R) C^{\prime}(w, t, R)\right) .
\end{gathered}
$$

Evidently, $R / 2 \leq|\Gamma(t, R)|$ for $R \in\left(0,2 d_{t}\right]$. Taking into account the latter inequality and the definitions of $C(w, t, R), C^{\prime}(w, t, R)$, we get for $t \in \Gamma$ and $R \in\left(0,2 d_{t}\right]$,

$$
\begin{aligned}
C(w, t, R) C^{\prime}(w, t, R) & \leq \frac{\left\|w \chi_{\Gamma(t, R)}\right\|_{X}\left\|\chi_{\Gamma(t, R)} / w\right\|_{X^{\prime}}}{|\Gamma(t, R)|} \cdot \frac{\left\|\chi_{\Gamma(t, R)}\right\|\left\|_{X}\right\| \chi_{\Gamma(t, R)} \|_{X^{\prime}}}{|\Gamma(t, R)|} \\
& \leq 4 B_{t, R}(w) B_{t, R}(1) .
\end{aligned}
$$

Therefore,

$$
\sup _{R \in\left(0,2 d_{t}\right]}\left(C(w, t, R) C^{\prime}(w, t, R)\right) \leq 4\left(\sup _{R \in\left(0,2 d_{t}\right]} B_{t, R}(w)\right)\left(\sup _{R \in\left(0,2 d_{t}\right]} B_{t, R}(1)\right) .
$$

From (3.8)-(3.10) it follows that

$$
\begin{aligned}
& \sup _{R>0}\left(C(w, t, R) C^{\prime}(w, t, R)\right) \leq 4\left(\sup _{R>0} B_{t, R}(w)\right)\left(\sup _{R>0} B_{t, R}(1)\right) \\
& \sup _{t \in \Gamma} \sup _{R>0}\left(C(w, t, R) C^{\prime}(w, t, R)\right) \leq 4\left(\sup _{t \in \Gamma} \sup _{R>0} B_{t, R}(w)\right)\left(\sup _{t \in \Gamma} \sup _{R>0} B_{t, R}(1)\right) .
\end{aligned}
$$

(a) If $w \in A_{X}(\Gamma, t)$ and $1 \in A_{X}(\Gamma, t)$, then (3.11) implies (3.6), but we have shown that (3.6) is equivalent to (3.2). Part (a) is proved. Part (b) is proved similarly by using (3.12) and the equivalence of (3.7) and (3.3).

Lemma 3.2. (a) If $w \in A_{X}(\Gamma, t)$ and $1 \in A_{X}(\Gamma, t)$, then $\log w \in B M O(\Gamma, t)$.

(b) If $w \in A_{X}(\Gamma)$ and $1 \in A_{X}(\Gamma)$, then $\log w \in B M O(\Gamma)$.

Proof. This statement is proved by analogy with [25. Lemma 1.6] (see also [3, Proposition 2.4]). Put $\Omega_{t}(R):=\Omega_{t}(\log w, R)$,

$$
\begin{aligned}
& \Gamma_{+}(t, R):=\left\{\tau \in \Gamma(t, R): \log w(\tau) \geq \Omega_{t}(R)\right\}, \\
& \Gamma_{-}(t, R):=\left\{\tau \in \Gamma(t, R): \log w(\tau)<\Omega_{t}(R)\right\} .
\end{aligned}
$$

Due to Jensen's inequality [33 p. 78],

$$
\begin{aligned}
& \exp \left(\frac{1}{|\Gamma(t, R)|} \int_{\Gamma(t, R)}\left|\log w(\tau)-\Omega_{t}(R)\right||d \tau|\right) \\
& \leq \frac{1}{|\Gamma(t, R)|} \int_{\Gamma_{+}(t, R)} \exp \left(\log w(\tau)-\Omega_{t}(R)\right)|d \tau| \\
& +\frac{1}{|\Gamma(t, R)|} \int_{\Gamma_{-}(t, R)} \exp \left(-\left(\log w(\tau)-\Omega_{t}(R)\right)\right)|d \tau| \\
& \leq \frac{1}{|\Gamma(t, R)|} \int_{\Gamma} \exp \left(\log w(\tau)-\Omega_{t}(R)\right) \chi_{\Gamma(t, R)}(\tau)|d \tau| \\
& +\frac{1}{|\Gamma(t, R)|} \int_{\Gamma} \exp \left(-\left(\log w(\tau)-\Omega_{t}(R)\right)\right) \chi_{\Gamma(t, R)}(\tau)|d \tau|
\end{aligned}
$$


Applying Hölder's inequality (see Lemma 2.2) to the first term on the right of (3.13), we get

$$
\begin{aligned}
& \frac{1}{|\Gamma(t, R)|} \int_{\Gamma} \exp \left(\log w(\tau)-\Omega_{t}(R)\right) \chi_{\Gamma(t, R)}(\tau)|d \tau| \\
& \leq\left\|\exp \left(\log w(\cdot)-\Omega_{t}(R)\right) \chi_{\Gamma(t, R)}(\cdot)\right\|_{X} \frac{\left\|\chi_{\Gamma(t, R)}\right\|_{X^{\prime}}}{|\Gamma(t, R)|} \\
& =e^{-\Omega_{t}(R)} \frac{\left\|\chi_{\Gamma(t, R)}\right\|_{X}\left\|\chi_{\Gamma(t, R)}\right\|_{X^{\prime}}}{|\Gamma(t, R)|}=C(w, t, R) .
\end{aligned}
$$

Analogously,

$$
\frac{1}{|\Gamma(t, R)|} \int_{\Gamma} \exp \left(-\left(\log w(\tau)-\Omega_{t}(R)\right)\right) \chi_{\Gamma(t, R)}(\tau)|d \tau| \leq C^{\prime}(w, t, R) .
$$

Combining (3.13)-(3.15), we see that for every $t \in \Gamma$ and $R>0$,

$$
\exp \left(\frac{1}{|\Gamma(t, R)|} \int_{\Gamma(t, R)}\left|\log w(\tau)-\Omega_{t}(R)\right||d \tau|\right) \leq C(w, t, R)+C^{\prime}(w, t, R) .
$$

Consequently,

$$
\begin{aligned}
& \|\log w\|_{*, t} \leq \log \left(\sup _{R>0} C(w, t, R)+\sup _{R>0} C^{\prime}(w, t, R)\right), \quad t \in \Gamma, \\
& \|\log w\|_{*} \leq \log \left(\sup _{t \in \Gamma} \sup _{R>0} C(w, t, R)+\sup _{t \in \Gamma} \sup _{R>0} C^{\prime}(w, t, R)\right) .
\end{aligned}
$$

Statement (a) follows from Lemma 3.1(a) and (3.16). Statement (b) follows from Lemma 3.1 (b) and (3.17).

For rearrangement-invariant Banach function spaces $X$, by using (2.1), we infer that $w \in A_{X}(\Gamma)$ implies $1 \in A_{X}(\Gamma)$. In that case, by Lemma 3.2(b), if $w \in A_{X}(\Gamma)$, then $\log w \in B M O(\Gamma)$. This result was obtained in [25. Lemma 1.6]. Note that for Lebesgue spaces $L^{p}, 1<p<\infty$, and Muckenhoupt classes $A_{p}(\Gamma)$ this fact is well known (see, e.g., [3, Proposition 2.4]).

\section{INDICES OF SUBMULTIPLICATIVE FUNCTIONS ASSOCIATED WITH WEIGHTED BANACH FUNCTION SPACES}

4.1. Submultiplicative functions and their indices. Following [3. Section 1.4], we say a function $\Phi:(0, \infty) \rightarrow(0, \infty]$ is regular if it is bounded in an open neighborhood of 1 . A function $\Phi:(0, \infty) \rightarrow(0, \infty]$ is said to be submultiplicative if

$$
\Phi(x y) \leq \Phi(x) \Phi(y) \text { for all } x, y \in(0, \infty) .
$$

It is easy to show that if $\Phi$ is regular and submultiplicative, then $\Phi$ is bounded away from zero in some open neighborhood of 1 . Moreover, in this case $\Phi(x)$ is finite for all $x \in(0, \infty)$. Given a regular and submultiplicative function $\Phi:(0, \infty) \rightarrow(0, \infty)$, one defines

$$
\alpha(\Phi):=\sup _{x \in(0,1)} \frac{\log \Phi(x)}{\log x}, \quad \beta(\Phi):=\inf _{x \in(1, \infty)} \frac{\log \Phi(x)}{\log x} .
$$

Clearly, $-\infty<\alpha(\Phi)$ and $\beta(\Phi)<\infty$. 
Theorem 4.1. (see [3, Theorem 1.13]). If $\Phi:(0, \infty) \rightarrow(0, \infty)$ is regular and submultiplicative, then

$$
\begin{aligned}
& \alpha(\Phi)=\lim _{x \rightarrow 0} \frac{\log \Phi(x)}{\log x}, \quad \beta(\Phi)=\lim _{x \rightarrow \infty} \frac{\log \Phi(x)}{\log x} \\
& \text { and }-\infty<\alpha(\Phi) \leq \beta(\Phi)<+\infty .
\end{aligned}
$$

The quantities $\alpha(\Phi)$ and $\beta(\Phi)$ are called the lower and upper indices of the regular and submultiplicative function $\Phi$, respectively.

4.2. Spirality indices. In this subsection we mainly follow [3, Ch. 1]. Fix $t \in \Gamma$. Suppose $\psi: \Gamma \backslash\{t\} \rightarrow(0, \infty)$ is a continuous function. Put

$$
F_{\psi}\left(R_{1}, R_{2}\right):=\max _{\tau \in \Gamma,|\tau-t|=R_{1}} \psi(\tau) / \min _{\tau \in \Gamma,|\tau-t|=R_{2}} \psi(\tau), \quad R_{1}, R_{2} \in\left(0, d_{t}\right] .
$$

By [3. Lemma 1.15], the function

$$
\left(W_{t} \psi\right)(x):= \begin{cases}\sup _{0<R \leq d_{t}} F_{\psi}(x R, R), & x \in(0,1], \\ \sup _{0<R \leq d_{t}} F_{\psi}\left(R, x^{-1} R\right), & x \in(1, \infty) .\end{cases}
$$

is submultiplicative. For $t \in \Gamma$, we have,

$$
\tau-t=|\tau-t| e^{i \arg (\tau-t)}, \quad \tau \in \Gamma \backslash\{t\},
$$

and the $\operatorname{argument} \arg (\tau-t)$ may be chosen to be a continuous function of $\tau \in \Gamma \backslash\{t\}$. Consider

$$
\eta_{t}(\tau):=e^{-\arg (\tau-t)} .
$$

Using the local Carleson constant $C_{\Gamma, t}$ instead of the global Carleson constant $C_{\Gamma}$, we can obtain the following local versions of [3] Theorem 1.10 and Lemma 1.17].

Lemma 4.2. If $\Gamma$ is locally a Carleson curve at $t \in \Gamma$, then

$$
\arg (\tau-t)=O(-\log |\tau-t|) \quad \text { as } \tau \rightarrow t .
$$

Lemma 4.3. If $\Gamma$ is locally a Carleson curve at $t \in \Gamma$, then the submultiplicative function $W_{t} \eta_{t}$ is regular.

Under the assumptions of Lemma 4.3 by Theorem 4.1 there exist the spirality indices

$$
\delta_{t}^{-}:=\alpha\left(W_{t} \eta_{t}\right), \quad \delta_{t}^{+}:=\beta\left(W_{t} \eta_{t}\right)
$$

of the curve $\Gamma$ at the point $t$ (see [3. Ch. 1]). If, in addition,

$$
\arg (\tau-t)=-\delta_{t} \log |\tau-t|+O(1) \quad \text { as } \quad \tau \rightarrow t,
$$

where $\delta_{t} \in \mathbb{R}$, then $\delta_{t}^{-}=\delta_{t}^{+}=\delta_{t}$ (see [3, Section 1.6]). Examples of Carleson curves with distinct spirality indices are also given there.

On a rectifiable Jordan curve we have $d \tau=e^{i \theta_{\Gamma}(\tau)}|d \tau|$ where $\theta_{\Gamma}(\tau)$ is the angle between the positively oriented real axis and the naturally oriented tangent of $\Gamma$ at $\tau$ (which exists almost everywhere). A rectifiable Jordan curve $\Gamma$ is said to be a Lyapunov curve if

$$
\left|\theta_{\Gamma}(\tau)-\theta_{\Gamma}(t)\right| \leq c|\tau-t|^{\mu}
$$

for some constants $c>0, \mu \in(0,1)$ and all $\tau, t \in \Gamma$. If $\theta_{\Gamma}$ is a function of bounded variation on $\Gamma$, then the curve $\Gamma$ is called a Radon curve (or a curve of bounded rotation). It is very well known that Lyapunov curves are smooth, but Radon 
curves may have at most countable set of corner points (or even cusps). All Lyapunov curves and Radon curves without cusps are Carleson curves (see, e.g., 28, Section 2.3]). The next statement is well known.

Proposition 4.4. If $\Gamma$ is either a Lyapunov Jordan curve or a Radon Jordan curve, then for every $t \in \Gamma$,

and, therefore, $\delta_{t}^{-}=\delta_{t}^{+}=0$.

$$
\arg (\tau-t)=O(1) \quad \text { as } \quad \tau \rightarrow t,
$$

4.3. Indices of powerlikeness. To investigate whether the weight $\left|(\tau-t)^{\gamma}\right| w(\tau)$ with arbitrary $\gamma \in \mathbb{C}$ belongs to the Muckenhoupt class $A_{p}(\Gamma)$, A. Böttcher and Yu. I. Karlovich introduced submultiplicative functions $V_{t} w$ and $V_{t}^{0} w$ associated with local properties of the weight $w$ at the point $t \in \Gamma$ (see [3, Ch. 3]).

Let $w$ be a weight on $\Gamma$ such that $\log w \in L^{1}(\Gamma(t, R))$ for every $R \in\left(0, d_{t}\right]$. Put

$$
H_{w}\left(R_{1}, R_{2}\right):=\exp \left(\Omega_{t}\left(\log w, R_{1}\right)\right) / \exp \left(\Omega_{t}\left(\log w, R_{2}\right)\right), \quad R_{1}, R_{2} \in\left(0, d_{t}\right] .
$$

Consider the functions

$$
\begin{aligned}
\left(V_{t} w\right)(x) & := \begin{cases}\sup _{0<R \leq d_{t}} H_{w}(x R, R), & x \in(0,1], \\
\sup _{0<R \leq d_{t}} H_{w}\left(R, x^{-1} R\right), & x \in(1, \infty),\end{cases} \\
\left(V_{t}^{0} w\right)(x) & :=\limsup _{R \rightarrow 0} H_{w}(x R, R), \quad x \in(0, \infty) .
\end{aligned}
$$

Lemma 4.5. The function $V_{t} w$ is submultiplicative. If $V_{t} w$ is regular, then $V_{t}^{0} w$ is regular and submultiplicative. Moreover, $\alpha\left(V_{t}^{0} w\right)=\alpha\left(V_{t} w\right)$ and $\beta\left(V_{t}^{0} w\right)=\beta\left(V_{t} w\right)$.

Lemma 4.6. If $\Gamma$ is locally a Carleson curve at $t \in \Gamma$ and $\log w \in B M O(\Gamma, t)$, then $V_{t} w$ and $V_{t}^{0} w$ are regular.

Lemmas 4.5 and 4.6 are proved by analogy with [3, Lemma 3.5(a)] and [3. Lemma 3.2(a)]. These statements are stated in 3 under the assumption that $\Gamma$ is a Carleson curve. But Lemma 4.5] is valid for arbitrary rectifiable curves $\Gamma$. Since Lemma 4.6 has a "local nature", we may use the "local" Carleson constant $C_{\Gamma, t}$ instead of the "global" Carleson constant $C_{\Gamma}$ in its proof. Under the assumptions of Lemma 4.6 in view of Theorem 4.1 for the weight $w$, there exist the indices of powerlikeness

$$
\mu_{t}:=\alpha\left(V_{t}^{0} w\right)=\alpha\left(V_{t} w\right), \quad \nu_{t}:=\beta\left(V_{t}^{0} w\right)=\beta\left(V_{t} w\right)
$$

at the point $t \in \Gamma$.

Obviously, for a power weight $w(\tau)=|\tau-t|^{\lambda_{t}}$, the indices of powerlikeness equal $\mu_{t}=\nu_{t}=\lambda_{t}$. Nontrivial examples of weights with distinct indices of powerlikeness are given in [3, Examples 3.24-3.28].

Lemma 4.7. (see 25] Lemma 2.4]). If $\Gamma$ is locally a Carleson curve at $t \in \Gamma$ and $\log w \in \operatorname{VMO}(\Gamma, t)$, then $\mu_{t}=\nu_{t}=0$.

4.4. Submultiplicative functions associated with weighted Banach function spaces. Let $\Gamma$ be a rectifiable Jordan curve and let $X$ be a Banach function space. Fix $t \in \Gamma$ and consider the portion of the curve $\Gamma$ in the annulus

$$
\Delta(t, R):=\Gamma(t, R) \backslash \Gamma(t, R / 2), \quad R>0 .
$$

Clearly,

$$
R / 2 \leq|\Delta(t, R)|, \quad R \in\left(0, d_{t}\right] .
$$


On the other hand, if $\Gamma$ is locally a Carleson curve at $t \in \Gamma$, then

$$
|\Delta(t, R)| \leq|\Gamma(t, R)| \leq C_{\Gamma, t} R, \quad R>0 .
$$

Suppose $w: \Gamma \rightarrow[0, \infty]$ is a weight such that $w \chi_{\Delta(t, R)} \in X$ and $\chi_{\Delta(t, R)} / w \in X^{\prime}$ for all $R \in\left(0, d_{t}\right]$. We denote

$$
G_{w}\left(R_{1}, R_{2}\right):=\frac{\left\|w \chi_{\Delta\left(t, R_{1}\right)}\right\| X\left\|\chi_{\Delta\left(t, R_{2}\right)} / w\right\|_{X^{\prime}}}{\left|\Delta\left(t, R_{2}\right)\right|}, \quad R_{1}, R_{2} \in\left(0, d_{t}\right] .
$$

Define the following functions (see [24, Section 5]):

$$
\begin{aligned}
& \left(Q_{t} w\right)(x):= \begin{cases}\sup _{0<R \leq d_{t}} G_{w}(x R, R), & x \in(0,1], \\
\sup _{0<R \leq d_{t}} G_{w}\left(R, x^{-1} R\right), & x \in(1, \infty),\end{cases} \\
& \left(Q_{t}^{0} w\right)(x):=\limsup _{R \rightarrow 0} G_{w}(x R, R), \quad x \in(0, \infty) .
\end{aligned}
$$

Lemma 4.8. The function $Q_{t} w$ is submultiplicative. If $Q_{t} w$ is regular, then $Q_{t}^{0} w$ is regular and submultiplicative. Moreover, $\alpha\left(Q_{t}^{0} w\right)=\alpha\left(Q_{t} w\right), \beta\left(Q_{t}^{0} w\right)=\beta\left(Q_{t} w\right)$.

Lemma 4.9. If $w \in A_{X}(\Gamma, t)$, then $Q_{t} w$ and $Q_{t}^{0} w$ are regular. Moreover,

$$
0 \leq \alpha\left(Q_{t} w\right)=\alpha\left(Q_{t}^{0} w\right) \leq \beta\left(Q_{t}^{0} w\right)=\beta\left(Q_{t} w\right) \leq 1 .
$$

These statements are proved in [24] Lemmas 5.1-5.2] and [24. Theorem 5.3], respectively, under the assumption that $X$ is rearrangement-invariant. But, actually, we did not use this assumption in those proofs. So we can literally repeat the proofs for arbitrary Banach function spaces.

\section{Relations BETWEen indices}

5.1. Case of general Banach function spaces. Let $\Gamma$ be a rectifiable Jordan curve, let $X$ be a Banach function space, and let $t \in \Gamma$.

Theorem 5.1. Suppose $w: \Gamma \rightarrow[0, \infty]$ is a weight such that $\log w \in L^{1}(\Gamma(t, R))$ for every $R \in\left(0, d_{t}\right]$ and $\psi: \Gamma \backslash\{t\} \rightarrow(0, \infty)$ is a continuous function. If the functions $V_{t} w$ and $W_{t} \psi$ are regular, then the function $V_{t}(\psi w)$ is regular too. Moreover,

$$
\begin{aligned}
& \alpha\left(V_{t} w\right)+\alpha\left(W_{t} \psi\right) \leq \alpha\left(V_{t}(\psi w)\right) \leq \min \left\{\alpha\left(V_{t} w\right)+\beta\left(W_{t} \psi\right), \beta\left(V_{t} w\right)+\alpha\left(W_{t} \psi\right)\right\} \\
& \beta\left(V_{t} w\right)+\beta\left(W_{t} \psi\right) \geq \beta\left(V_{t}(\psi w)\right) \geq \max \left\{\alpha\left(V_{t} w\right)+\beta\left(W_{t} \psi\right), \beta\left(V_{t} w\right)+\alpha\left(W_{t} \psi\right)\right\} .
\end{aligned}
$$

This statement is proved similarly to [3, Lemma 3.17].

Theorem 5.2. Suppose $w: \Gamma \rightarrow[0, \infty]$ is a weight such that $w \chi_{\Delta(t, R)} \in X$ and $\chi_{\Delta(t, R)} / w \in X^{\prime}$ for every $R \in\left(0, d_{t}\right]$ and $\psi: \Gamma \backslash\{t\} \rightarrow(0, \infty)$ is a continuous function. If the functions $Q_{t} w$ and $W_{t} \psi$ are regular, then the function $Q_{t}(\psi w)$ is regular too. Moreover,

$$
\begin{aligned}
& \alpha\left(Q_{t} w\right)+\alpha\left(W_{t} \psi\right) \leq \alpha\left(Q_{t}(\psi w)\right) \leq \min \left\{\alpha\left(Q_{t} w\right)+\beta\left(W_{t} \psi\right), \beta\left(Q_{t} w\right)+\alpha\left(W_{t} \psi\right)\right\}, \\
& \beta\left(Q_{t} w\right)+\beta\left(W_{t} \psi\right) \geq \beta\left(Q_{t}(\psi w)\right) \geq \max \left\{\alpha\left(Q_{t} w\right)+\beta\left(W_{t} \psi\right), \beta\left(Q_{t} w\right)+\alpha\left(W_{t} \psi\right)\right\} .
\end{aligned}
$$

This theorem is proved in [24. Theorem 5.8] for rearrangement-invariant Banach function spaces. The proof given there does not use the rearrangement-invariant property of the space, so it works for an arbitrary Banach function space. 
Lemma 5.3. If $\Gamma$ is locally a Carleson curve at $t \in \Gamma$ and $\log w \in B M O(\Gamma, t)$, then for every $R \in\left(0, d_{t}\right]$,

$$
\exp \left(\Omega_{t}(\log w, R)\right) \leq \frac{C_{t}}{|\Delta(t, R)|} \int_{\Delta(t, R)} w(\tau)|d \tau|
$$

where $C_{t}:=\exp \left(2 C_{\Gamma, t}\|\log w\|_{*, t}\right)<\infty$.

The proof is actually given in [3, Lemma 3.2(b)].

Theorem 5.4. Let $\Gamma$ be locally a Carleson curve at $t \in \Gamma$ and let $w: \Gamma \rightarrow[0, \infty]$ be a weight such that $w \chi_{\Delta(t, R)} \in X, \chi_{\Delta(t, R)} / w \in X^{\prime}$ for every $R \in\left(0, d_{t}\right]$ and $\log w \in B M O(\Gamma, t)$. If $Q_{t} w$ and $Q_{t} 1$ are regular, then

$$
\alpha\left(Q_{t} w\right) \leq \mu_{t}+\beta\left(Q_{t} 1\right), \quad \nu_{t}+\alpha\left(Q_{t} 1\right) \leq \beta\left(Q_{t} w\right) .
$$

Proof. The proof is developed by analogy with [25. Theorem 2.6]. From Lemma 5.3 and Hölder's inequality (see Lemma [2.2) we see that for every $R \in\left(0, d_{t}\right]$,

$$
\begin{aligned}
\exp \left(\Omega_{t}(\log w, R)\right) & \leq C_{t} \frac{\left\|w \chi_{\Delta(t, R)}\right\|_{X}\left\|\chi_{\Delta(t, R)}\right\|_{X^{\prime}}}{|\Delta(t, R)|}, \\
\exp \left(-\Omega_{t}(\log w, R)\right) & \leq C_{t} \frac{\left\|\chi_{\Delta(t, R)}\right\| X\left\|\chi_{\Delta(t, R)} / w\right\|_{X^{\prime}}}{|\Delta(t, R)|} .
\end{aligned}
$$

From (5.2) and (5.3) it follows that for $x \in(0,1]$ and $R \in\left(0, d_{t}\right]$,

$$
\begin{aligned}
& H_{w}(x R, R)=\exp \left(\Omega_{t}(\log w, x R)\right) \exp \left(-\Omega_{t}(\log w, R)\right) \\
& \leq C_{t}^{2} \frac{\left\|w \chi_{\Delta(t, x R)}\right\|_{X}\left\|\chi_{\Delta(t, R)} / w\right\|_{X^{\prime}}}{|\Delta(t, R)|} \cdot \frac{\left\|\chi_{\Delta(t, R)}\right\|_{X}\left\|\chi_{\Delta(t, x R)}\right\|_{X^{\prime}}}{|\Delta(t, x R)|} \\
& =C_{t}^{2} G_{w}(x R, R) G_{1}(R, x R) .
\end{aligned}
$$

Then, taking the supremum over all $R \in\left(0, d_{t}\right]$, we obtain for $x \in(0,1]$,

$$
\left(V_{t} w\right)(x) \leq C_{t}\left(Q_{t} w\right)(x)\left(Q_{t} 1\right)\left(x^{-1}\right) .
$$

Analogously, for $x \in(1, \infty)$ and $R \in\left(0, d_{t}\right]$,

$$
H_{w}\left(R, x^{-1} R\right) \leq C_{t}^{2} G_{w}\left(R, x^{-1} R\right) G_{1}\left(x^{-1} R, R\right) .
$$

Taking the supremum over all $R \in\left(0, d_{t}\right]$, we arrive at (5.5) for $x \in(1, \infty)$. By Lemmas 4.5 4.6 the function $V_{t} w$ is regular and submultiplicative. By Lemma 4.8 the functions $Q_{t} w$ and $Q_{t} 1$ are submultiplicative, they are regular, due to the assumption of the theorem. Therefore, in view of Theorem 4.1 the indices $\alpha\left(Q_{t} w\right), \beta\left(Q_{t} w\right)$; $\alpha\left(Q_{t} 1\right), \beta\left(Q_{t} 1\right)$; and $\alpha\left(V_{t} w\right), \beta\left(V_{t} w\right)$ exist and are well defined.

From (5.5) it follows that

$$
\begin{array}{ll}
\frac{\log \left(V_{t} w\right)(x)}{\log x} \geq \frac{\log C_{t}^{2}}{\log x}+\frac{\log \left(Q_{t} w\right)(x)}{\log x}-\frac{\log \left(Q_{t} 1\right)\left(x^{-1}\right)}{\log x^{-1}}, \quad x \in(0,1], \\
\frac{\log \left(V_{t} w\right)(x)}{\log x} \leq \frac{\log C_{t}^{2}}{\log x}+\frac{\log \left(Q_{t} w\right)(x)}{\log x}-\frac{\log \left(Q_{t} 1\right)\left(x^{-1}\right)}{\log x^{-1}}, \quad x \in(1, \infty) .
\end{array}
$$

Passing to the limit in the latter inequalities as $x \rightarrow 0$ (respectively, as $x \rightarrow \infty$ ), we obtain, respectively,

$$
\mu_{t}=\alpha\left(V_{t} w\right) \geq \alpha\left(Q_{t} w\right)-\beta\left(Q_{t} 1\right), \quad \nu_{t}=\beta\left(V_{t} w\right) \leq \beta\left(Q_{t} w\right)-\alpha\left(Q_{t} 1\right) .
$$

So, we arrive at (5.1). 
Theorem 5.5. If $w \in A_{X}(\Gamma, t)$ and $1 \in A_{X}(\Gamma, t)$, then

$$
\begin{aligned}
& \alpha\left(Q_{t} 1\right)+\mu_{t} \leq \alpha\left(Q_{t} w\right) \leq \min \left\{\alpha\left(Q_{t} 1\right)+\nu_{t}, \beta\left(Q_{t} 1\right)+\mu_{t}\right\}, \\
& \beta\left(Q_{t} 1\right)+\nu_{t} \geq \beta\left(Q_{t} w\right) \geq \max \left\{\alpha\left(Q_{t} 1\right)+\nu_{t}, \beta\left(Q_{t} 1\right)+\mu_{t}\right\} .
\end{aligned}
$$

Proof. The idea of the proof is borrowed from [25. Theorems 2.6 and 2.7]. From Lemmas 4.8 4.9 it follows that the functions $Q_{t} w$ and $Q_{t} 1$ are regular and submultiplicative. On the other hand, by Lemma B.2(a), $\log w \in B M O(\Gamma, t)$. Therefore, by Lemmas 4.5 4.6. the function $V_{t} w$ is regular and submultiplicative. Thus, all the indices

$$
\alpha\left(Q_{t} 1\right), \quad \beta\left(Q_{t} 1\right), \quad \alpha\left(Q_{t} w\right), \quad \beta\left(Q_{t} w\right), \quad \mu_{t}=\alpha\left(V_{t} w\right), \quad \nu_{t}=\beta\left(V_{t} w\right)
$$

are well defined. By Theorem 5.4

$$
\alpha\left(Q_{t} w\right) \leq \mu_{t}+\beta\left(Q_{t} 1\right), \quad \nu_{t}+\alpha\left(Q_{t} 1\right) \leq \beta\left(Q_{t} w\right) .
$$

If $1 \in A_{X}(\Gamma, t)$, then from the lattice property it follows that for every $R>0$,

$$
\begin{aligned}
\frac{1}{R}\left\|\chi_{\Delta(t, R)}\right\|_{X}\left\|\chi_{\Delta(t, R)}\right\|_{X^{\prime}} & \leq \frac{1}{R}\left\|\chi_{\Gamma(t, R)}\right\|_{X}\left\|\chi_{\Gamma(t, R)}\right\|_{X^{\prime}} \\
& \leq \sup _{R>0} B_{t, R}(1)=: B_{t}(1) .
\end{aligned}
$$

Combining (5.10) and (4.2), we arrive at

$$
\left\|\chi_{\Delta(t, R)}\right\|_{X}\left\|\chi_{\Delta(t, R)}\right\|_{X^{\prime}} \leq 2 B_{t}(1)|\Delta(t, R)|, \quad R \in\left(0, d_{t}\right] .
$$

Then we have for $x \in(0,1]$,

$$
\begin{aligned}
\frac{1}{G_{1}(R, x R)} & =\frac{|\Delta(t, x R)|}{\left\|\chi_{\Delta(t, R)}\right\|_{X}\left\|\chi_{\Delta(t, x R)}\right\|_{X^{\prime}}} \geq \frac{\left\|\chi_{\Delta(t, x R)}\right\|_{X}\left\|\chi_{\Delta(t, R)}\right\|_{X^{\prime}}}{\left(2 B_{t}(1)\right)^{2}|\Delta(t, R)|} \\
& =\left(2 B_{t}(1)\right)^{-2} G_{1}(x R, R) .
\end{aligned}
$$

Analogously, we deduce that for $x \in(1, \infty)$,

$$
\frac{1}{G_{1}\left(x^{-1} R, R\right)} \geq\left(2 B_{t}(1)\right)^{-2} G_{1}\left(R, x^{-1} R\right) .
$$

From (5.4) and (5.11) we obtain for $x \in(0,1]$,

$$
\begin{aligned}
\left(2 B_{t}(1)\right)^{-2} G_{1}(x R, R) & \leq \frac{1}{G_{1}(R, x R)} \leq C_{t}^{2} \frac{G_{w}(x R, R)}{H_{w}(x R, R)} \\
& =C_{t}^{2} G_{w}(x R, R) H_{w}(R, x R) .
\end{aligned}
$$

Similarly, from (5.6) and (5.12) we obtain for $x \in(1, \infty)$,

$$
\left(2 B_{t}(1)\right)^{-2} G_{1}\left(R, x^{-1} R\right) \leq C_{t}^{2} G_{w}(x R, R) H_{w}\left(x^{-1} R, R\right) .
$$

Taking the supremum over $R \in\left(0, d_{t}\right]$ in (5.13) and (5.14), we get

$$
\left(Q_{t} 1\right)(x) \leq\left(2 C_{t} B_{t}(1)\right)^{2}\left(Q_{t} w\right)(x)\left(V_{t} w\right)\left(x^{-1}\right), \quad x \in(0, \infty) .
$$

From this inequality it follows that for $x \in(0,1]$,

$$
\frac{\log \left(Q_{t} 1\right)(x)}{\log x} \geq \frac{\log \left(2 C_{t} B_{t}(1)\right)^{2}}{\log x}+\frac{\log \left(Q_{t} w\right)(x)}{\log x}-\frac{\log \left(V_{t} w\right)\left(x^{-1}\right)}{\log x^{-1}}
$$

and, analogously, for $x \in(1, \infty)$,

$$
\frac{\log \left(Q_{t} 1\right)(x)}{\log x} \leq \frac{\log \left(2 C_{t} B_{t}(1)\right)^{2}}{\log x}+\frac{\log \left(Q_{t} w\right)(x)}{\log x}-\frac{\log \left(V_{t} w\right)\left(x^{-1}\right)}{\log x^{-1}} .
$$


ALEXEI YU. KARLOVICH

Passing to the limit in (5.15) as $x \rightarrow 0$ and in (5.16) as $x \rightarrow \infty$, we obtain, respectively,

$$
\alpha\left(Q_{t} 1\right) \geq \alpha\left(Q_{t} w\right)-\beta\left(V_{t} w\right), \quad \beta\left(Q_{t} 1\right) \leq \beta\left(Q_{t} w\right)-\alpha\left(V_{t} w\right) .
$$

By Lemma 3.11 (a), there exist constants $C_{1}(t), C_{2}(t)>0$ such that for every $R>0$,

$$
\begin{aligned}
& \exp \left(-\Omega_{t}(\log w, R)\right) \frac{\left\|w \chi_{\Gamma(t, R)}\right\|_{X}\left\|\chi_{\Gamma(t, R)}\right\|_{X^{\prime}}}{|\Gamma(t, R)|} \leq C_{1}(t), \\
& \exp \left(\Omega_{t}(\log w, R)\right) \frac{\left\|\chi_{\Gamma(t, R)}\right\|_{X}\left\|\chi_{\Gamma(t, R)} / w\right\|_{X^{\prime}}}{|\Gamma(t, R)|} \leq C_{2}(t) .
\end{aligned}
$$

On the other hand, from the lattice property, the Hölder inequality (see Lemma 2.2), (3.1) and (4.2) it follows that for $R \in\left(0, d_{t}\right]$,

$$
\begin{aligned}
\frac{|\Gamma(t, R)|}{\left\|\chi_{\Gamma(t, R)}\right\|_{X^{\prime}}} & \leq \frac{|\Gamma(t, R)|}{\left\|\chi_{\Delta(t, R)}\right\|_{X^{\prime}}}=\frac{|\Gamma(t, R)| \cdot\left\|\chi_{\Delta(t, R)}\right\|_{X}}{\left\|\chi_{\Delta(t, R)}\right\|_{X}\left\|\chi_{\Delta(t, R)}\right\|_{X^{\prime}}} \\
& \leq \frac{|\Gamma(t, R)|}{|\Delta(t, R)|}\left\|_{\Delta(t, R)}\right\|_{X} \leq \frac{C_{\Gamma, t} R}{R / 2}\left\|\chi_{\Delta(t, R)}\right\|_{X} \\
& =2 C_{\Gamma, t}\left\|\chi_{\Delta(t, R)}\right\|_{X} .
\end{aligned}
$$

Analogously, for $R \in\left(0, d_{t}\right]$,

$$
\frac{|\Gamma(t, R)|}{\left\|\chi_{\Gamma(t, R)}\right\|_{X}} \leq 2 C_{\Gamma, t}\left\|\chi_{\Delta(t, R)}\right\|_{X^{\prime}}
$$

From (5.18) - (5.21) and the lattice property it follows that for $R \in\left(0, d_{t}\right]$ and $x \in(0,1]$,

$$
\begin{aligned}
& G_{w}(x R, R)=\frac{\left\|w \chi_{\Delta(t, x R)}\right\|_{X}\left\|\chi_{\Delta(t, R)} / w\right\|_{X^{\prime}}}{|\Delta(t, R)|} \\
& \leq \frac{\left\|w \chi_{\Gamma(t, x R)}\right\| x\left\|\chi_{\Gamma(t, R)} / w\right\|_{X^{\prime}}}{|\Delta(t, R)|} \\
& \leq \frac{C_{1}(t) C_{2}(t)}{|\Delta(t, R)|} \exp \left(\Omega_{t}(\log w, x R)\right) \exp \left(-\Omega_{t}(\log w, R)\right) \\
& \times \frac{|\Gamma(t, x R)|}{\left\|\chi_{\Gamma(t, x R)}\right\|_{X^{\prime}}} \cdot \frac{|\Gamma(t, R)|}{\left\|\chi_{\Gamma(t, R)}\right\|_{X}} \\
& \leq\left(2 C_{\Gamma, t}\right)^{2} C_{1}(t) C_{2}(t) H_{w}(x R, R) \frac{\left\|\chi_{\Delta(t, x R)}\right\|_{X}\left\|\chi_{\Delta(t, R)}\right\|_{X^{\prime}}}{|\Delta(t, R)|} \\
& =\left(2 C_{\Gamma, t}\right)^{2} C_{1}(t) C_{2}(t) H_{w}(x R, R) G_{1}(x R, R)
\end{aligned}
$$

and, similarly, for $R \in\left(0, d_{t}\right]$ and $x \in(1, \infty)$,

$$
G_{w}\left(R, x^{-1} R\right) \leq\left(2 C_{\Gamma, t}\right)^{2} C_{1}(t) C_{2}(t) H_{w}\left(R, x^{-1} R\right) G_{1}\left(R, x^{-1} R\right) .
$$

Taking the supremum over all $R \in\left(0, d_{t}\right]$ in (5.22) and (5.23), we obtain

$$
\left(Q_{t} w\right)(x) \leq\left(2 C_{\Gamma, t}\right)^{2} C_{1}(t) C_{2}(t)\left(V_{t} w\right)(x)\left(Q_{t} 1\right)(x), \quad x \in(0, \infty) .
$$

Therefore,

$$
\alpha\left(Q_{t} w\right) \geq \alpha\left(V_{t} w\right)+\alpha\left(Q_{t} 1\right), \quad \beta\left(Q_{t} w\right) \leq \beta\left(V_{t} w\right)+\beta\left(Q_{t} 1\right) .
$$

Combining (5.9), (5.17), and (5.24), we arrive at (5.7)-(5.8). 
If $X$ is a rearrangement-invariant Banach function space, then from (2.1) it follows that the conditions $1 \in A_{X}(\Gamma, t)$ and $1 \in A_{X}(\Gamma)$ are equivalent to (3.1) and (1.1), respectively. Hence, $w \in A_{X}(\Gamma, t)$ implies $1 \in A_{X}(\Gamma, t)$ whenever $X$ is rearrangement-invariant. This property allows us to simplify the formulation of Theorem [5.5 for rearrangement-invariant Banach function spaces (see [25], Theorems 2.6 and 2.7]).

Note that $\alpha\left(Q_{t} 1\right)$ and $\beta\left(Q_{t} 1\right)$ can be considered as a generalization of the Zippin (fundamental) indices $p_{X}$ and $q_{X}$ of a rearrangement-invariant Banach function space $X$ [53]. If $X$ is rearrangement-invariant, then $\alpha\left(Q_{t} 1\right)=p_{X}$ and $\beta\left(Q_{t} 1\right)=$ $q_{X}$ (see [24] Theorem 5.4]). On the other hand, the Zippin indices for an Orlicz space $L^{\varphi}$ coincide with the reciprocals of the Matuszewska-Orlicz indices, which control the growth of the Young function $\varphi$ (see, e.g., [39] and the references given there). The notion of Matuszewska-Orlicz indices of Orlicz spaces was extended to the case of Musielak-Orlicz spaces in [18, 19. Remind that Orlicz spaces are always rearrangement-invariant, but Musielak-Orlicz spaces are not rearrangementinvariant, in general.

5.2. Case of Nakano spaces. Suppose $\Gamma$ is a rectifiable Jordan curve. Assume that $p: \Gamma \rightarrow(1, \infty)$ is a continuous function. Then

$$
1<p_{*}:=\min _{t \in \Gamma} p(t) \leq \max _{t \in \Gamma} p(t):=p^{*}<\infty,
$$

due to the compactness of $\Gamma$. We will say that a continuous function $p: \Gamma \rightarrow(1, \infty)$ belongs to the class $\mathcal{P}_{t}$ if there is a constant $A_{t}>0$ such that

$$
|p(\tau)-p(t)| \leq \frac{A_{t}}{-\log |\tau-t|} \quad \text { for all } \quad \tau \in \Gamma(t, 1 / 2) .
$$

The class of all continuous functions $p: \Gamma \rightarrow(1, \infty)$ such that $p \in \mathcal{P}_{t}$ for every $t \in \Gamma$ and

$$
\sup _{t \in \Gamma} A_{t}=: A<\infty
$$

is denoted by $\mathcal{P}$. Clearly, $\mathcal{P} \subset \mathcal{P}_{t}$ for every $t \in \Gamma$.

The class $\mathcal{P}$ plays a very important role in questions on the boundedness of maximal functions and singular integrals on (weighted) Nakano spaces (see [10, 30, [46], the references therein, and also Theorem 6.2).

Proposition 5.6. A function $p$ belongs to $\mathcal{P}_{t}$ (respectively, to $\mathcal{P}$ ) if and only if the function $p^{\prime}(\tau):=p(\tau) /(p(\tau)-1)$ belongs to $\mathcal{P}_{t}$ (respectively, to $\left.\mathcal{P}\right)$.

Proof. The statement immediately follows from the obvious inequality

$$
\left|p^{\prime}(\tau)-p^{\prime}(t)\right|=\left|\frac{p(\tau)-p(t)}{(p(\tau)-1)(p(t)-1)}\right| \leq \frac{|p(\tau)-p(t)|}{\left(p_{*}-1\right)^{2}}, \quad \tau, t \in \Gamma,
$$

and the reflexive relation $\left(p^{\prime}\right)^{\prime}=p$.

Lemma 5.7. Let $\Gamma$ be locally a Carleson curve at $t \in \Gamma$ and $p \in \mathcal{P}_{t}$. Then there exist constants $M_{1}(t), M_{2}(t), C_{1}(t), C_{2}(t) \in(0, \infty)$ such that

$$
\begin{aligned}
&\left\|\chi_{\Delta(t, R)}\right\|_{L^{p(\cdot)}} \geq M_{1}(t) R^{1 / p(t)} \quad \text { for all } \quad R \in\left(0, C_{1}(t)\right), \\
&\left\|\chi_{\Gamma(t, R)}\right\|_{L^{p(\cdot)}} \leq M_{2}(t) R^{1 / p(t)} \quad \text { for all } \quad R \in\left(0, C_{2}(t)\right) .
\end{aligned}
$$


Proof. From (5.26) it follows that

$$
-p(t)-\frac{A_{t}}{-\log |\tau-t|} \leq-p(\tau) \leq-p(t)+\frac{A_{t}}{-\log |\tau-t|}, \quad \tau \in \Gamma(t, 1 / 2) .
$$

Since $|\tau-t| \leq R$ for $\tau \in \Gamma(t, R)$, we have

$$
\frac{A_{t}}{-\log |\tau-t|} \leq \frac{A_{t}}{-\log R}, \quad \tau \in \Gamma(t, R), \quad R \in(0,1 / 2)
$$

From (5.29) and (5.30) we get for $\tau \in \Gamma(t, R)$ and $R \in(0,1 / 2)$,

$$
-p(t)+\frac{A_{t}}{\log R} \leq-p(\tau) \leq-p(t)-\frac{A_{t}}{\log R} .
$$

For $R \in\left(0, e^{-A_{t}}\right)$, taking into account that $p(t) \in(1, \infty)$, we obtain

$$
p(t)+\frac{A_{t}}{\log R}=(p(t)-1)+\left(1+\frac{A_{t}}{\log R}\right)>p(t)-1>0 .
$$

From (5.31) we get for $\lambda \in(0,1]$ and $R \in\left(0, \min \left\{1 / 2, e^{-A_{t}}\right\}\right)$,

$$
\begin{aligned}
\exp \left(-\left[p(t)+\frac{A_{t}}{\log R}\right] \log \lambda\right) & \leq \exp (-p(\tau) \log \lambda) \\
& \leq \exp \left(-\left[p(t)-\frac{A_{t}}{\log R}\right] \log \lambda\right) .
\end{aligned}
$$

Analogously, for $\lambda \in(1, \infty)$ and $R \in\left(0, \min \left\{1 / 2, e^{-A_{t}}\right\}\right)$,

$$
\begin{aligned}
\exp \left(-\left[p(t)-\frac{A_{t}}{\log R}\right] \log \lambda\right) & \leq \exp (-p(\tau) \log \lambda) \\
& \leq \exp \left(-\left[p(t)+\frac{A_{t}}{\log R}\right] \log \lambda\right) .
\end{aligned}
$$

Let us prove (5.27). From the first inequality in (5.33) and (4.2) it follows that for $\lambda \in(0,1]$ and $R \in\left(0, \min \left\{1 / 2, e^{-A_{t}}, d_{t}\right\}\right)$,

$$
\begin{aligned}
m\left(\chi_{\Delta(t, R)} / \lambda, p\right) & =\int_{\Delta(t, R)} \exp (-p(\tau) \log \lambda)|d \tau| \\
& \geq \exp \left(-\left[p(t)+\frac{A_{t}}{\log R}\right] \log \lambda\right)|\Delta(t, R)| \\
& \geq \exp \left(\log \frac{R}{2}-\left[p(t)+\frac{A_{t}}{\log R}\right] \log \lambda\right) .
\end{aligned}
$$

Put $C_{1}(t):=\min \left\{1 / 2, e^{-A_{t}}, d_{t}\right\}$. Therefore, taking into account (5.32), we obtain for $R \in\left(0, C_{1}(t)\right)$,

$$
\begin{aligned}
& \left\{\lambda \in(0,1]: \quad m\left(\chi_{\Delta(t, R)} / \lambda, p\right) \leq 1\right\} \\
& \subset\left\{\lambda \in(0,1]: \quad \log \frac{R}{2}-\left[p(t)+\frac{A_{t}}{\log R}\right] \log \lambda \leq 0\right\} \\
& =\left\{\lambda: \quad \exp \left(\frac{\log (R / 2)}{p(t)+A_{t} / \log R}\right) \leq \lambda \leq 1\right\} .
\end{aligned}
$$

Thus, for $R \in\left(0, C_{1}(t)\right)$,

(5.35) $N_{1}:=\inf \left\{\lambda \in(0,1]: \quad m\left(\chi_{\Delta(t, R)} / \lambda, p\right) \leq 1\right\} \geq \exp \left(\frac{\log (R / 2)}{p(t)+A_{t} / \log R}\right)$. 
Analogously, from the first inequality in (5.34) we obtain

$$
\left\{\lambda \in(1, \infty): \quad m\left(\chi_{\Delta(t, R)} / \lambda, p\right) \leq 1\right\} \subset(1, \infty)
$$

because

$$
\exp \left(\frac{\log (R / 2)}{p(t)-A_{t} / \log R}\right)<1 \quad \text { for } \quad R \in\left(0, C_{1}(t)\right) .
$$

Thus, for $R \in\left(0, C_{1}(t)\right)$,

$$
N_{2}:=\inf \left\{\lambda \in(1, \infty): \quad m\left(\chi_{\Delta(t, R)} / \lambda, p\right) \leq 1\right\} \geq 1 .
$$

From (5.35) - (5.37) we obtain for $R \in\left(0, C_{1}(t)\right)$,

$$
\begin{aligned}
& \left\|\chi_{\Delta(t, R)}\right\|_{L^{p(\cdot)}}=\inf \left\{\lambda>0: \quad m\left(\chi_{\Delta(t, R)} / \lambda, p\right) \leq 1\right\}=\min \left\{N_{1}, N_{2}\right\} \\
& \geq \min \left\{1, \exp \left(\frac{\log (R / 2)}{p(t)+A_{t} / \log R}\right)\right\}=\exp \left(\frac{\log (R / 2)}{p(t)+A_{t} / \log R}\right) .
\end{aligned}
$$

From (5.32) it follows that for $R \in\left(0, C_{1}(t)\right)$,

$$
\frac{\log \frac{R}{2}}{p(t)+\frac{A_{t}}{\log R}}-\frac{\log \frac{R}{2}}{p(t)}=\frac{-A_{t}+A_{t} \frac{\log 2}{\log R}}{\left(p(t)+\frac{A_{t}}{\log R}\right) p(t)} \geq \frac{-A_{t}+A_{t} \frac{\log 2}{\log R}}{(p(t)-1) p(t)} \geq \frac{A_{t}+\log 2}{(1-p(t)) p(t)} .
$$

From the latter inequality we deduce that

$$
\begin{aligned}
& \exp \left(\frac{\log (R / 2)}{p(t)+A_{t} / \log R}\right)=\exp \left(\frac{\log (R / 2)}{p(t)+A_{t} / \log R}-\frac{\log (R / 2)}{p(t)}\right)\left(\frac{R}{2}\right)^{1 / p(t)} \\
& \geq \exp \left(\frac{A_{t}+\log 2}{(1-p(t)) p(t)}-\frac{\log 2}{p(t)}\right) R^{1 / p(t)}
\end{aligned}
$$

Combining (5.38) and (5.39), we arrive at (5.27) with

$$
C_{1}(t):=\min \left\{1 / 2, e^{-A_{t}}, d_{t}\right\}, \quad M_{1}(t):=\exp \left(\frac{A_{t}+\log 2}{(1-p(t)) p(t)}-\frac{\log 2}{p(t)}\right) .
$$

Taking into account (3.1), one can prove that (5.28) is valid with

$$
C_{2}(t):=\min \left\{1 / 2,1 / C_{\Gamma, t}, e^{-A_{t}}, d_{t}\right\}, \quad M_{2}(t):=\exp \left(\frac{A_{t}}{(p(t))^{2}}+\frac{\log C_{\Gamma, t}}{p(t)}\right) .
$$

The proof of (5.28) is similar to the proof of (5.27) and it is omitted.

Lemma 5.8. Suppose $\Gamma$ is locally a Carleson curve at $t \in \Gamma$ and $p \in \mathcal{P}_{t}$. Then $1 \in A_{L^{p(\cdot)}}(\Gamma, t)$ and

$$
\alpha\left(Q_{t} 1\right)=\beta\left(Q_{t} 1\right)=1 / p(t) .
$$

Proof. From Lemma 5.7 we deduce that there exist constants $C_{i}(t), M_{i}(t)(i=1,2)$ such that

$$
\begin{aligned}
&\left\|\chi_{\Delta(t, R)}\right\|_{L^{p(\cdot)}} \geq M_{1}(t) R^{1 / p(t)} \quad \text { for all } \quad R \in\left(0, C_{1}(t)\right), \\
&\left\|\chi_{\Gamma(t, R)}\right\|_{L^{p(\cdot)}} \leq M_{2}(t) R^{1 / p(t)} \quad \text { for all } \quad R \in\left(0, C_{2}(t)\right),
\end{aligned}
$$

By Proposition [5.6 $p^{\prime} \in \mathcal{P}_{t}$. Analogously, applying Lemma [5.7 to $L^{p^{\prime}(\cdot)}$ and taking into account that the latter space coincide with $\left(L^{p(\cdot)}\right)^{\prime}$ up to the equivalence of the 
norms (see Lemma 2.4), we infer that there exist constants $C_{i}^{\prime}(t), M_{i}^{\prime}(t)(i=1,2)$ such that

$$
\begin{aligned}
\left\|\chi_{\Delta(t, R)}\right\|_{\left(L^{p(\cdot)}\right)^{\prime}} & \geq M_{1}^{\prime}(t) R^{1 / p^{\prime}(t)} \quad \text { for all } \quad R \in\left(0, C_{1}^{\prime}(t)\right), \\
\left\|\chi_{\Gamma(t, R)}\right\|_{\left(L^{p(\cdot)}\right)^{\prime}} \leq M_{2}^{\prime}(t) R^{1 / p^{\prime}(t)} & \text { for all } \quad R \in\left(0, C_{2}^{\prime}(t)\right) .
\end{aligned}
$$

From (5.42), (5.44) it follows that for $R \in\left(0, \min \left\{C_{2}(t), C_{2}^{\prime}(t)\right\}\right)$,

$$
\begin{aligned}
B_{t, R}(1) & =\frac{1}{R}\left\|\chi_{\Gamma(t, R)}\right\|_{L^{p(\cdot)}}\left\|\chi_{\Gamma(t, R)}\right\|_{\left(L^{p(\cdot)}\right)^{\prime}} \\
& \leq \frac{1}{R} M_{2}(t) M_{2}^{\prime}(t) R^{1 / p(t)} R^{1 / p^{\prime}(t)}=M_{2}(t) M_{2}^{\prime}(t) .
\end{aligned}
$$

On the other hand, for $R \geq \min \left\{C_{2}(t), C_{2}^{\prime}(t)\right\}$,

$$
B_{t, R}(1)=\frac{1}{R}\left\|\chi_{\Gamma(t, R)}\right\|_{L^{p(\cdot)}}\left\|\chi_{\Gamma(t, R)}\right\|_{\left(L^{p(\cdot)}\right)^{\prime}} \leq \frac{\|1\|_{L^{p(\cdot)}}\|1\|_{\left(L^{p(\cdot)}\right)^{\prime}}}{\min \left\{C_{2}(t), C_{2}^{\prime}(t)\right\}} .
$$

From (5.45) and (5.46) it follows that

$$
\sup _{R>0} B_{t, R}(1) \leq \max \left\{M_{2}(t) M_{2}^{\prime}(t), \frac{\|1\|_{L^{p(\cdot)}}\|1\|_{\left(L^{p(\cdot)}\right)^{\prime}}}{\min \left\{C_{2}(t), C_{2}^{\prime}(t)\right\}}\right\}<\infty .
$$

Thus, $1 \in A_{L^{p(\cdot)}}(\Gamma, t)$.

Put $C(t):=\min \left\{C_{1}(t), C_{2}(t), C_{1}^{\prime}(t), C_{2}^{\prime}(t)\right\}$. From (5.42), (5.44), (4.2), and the lattice property we obtain for $x \in(0, \infty)$ and $R \in(0, C(t) \min \{1,1 / x\})$,

$$
\begin{aligned}
G_{1}(x R, R) & :=\frac{\left\|\chi_{\Delta(t, x R)}\right\|_{L^{p(\cdot)} \|}\left\|\chi_{\Delta(t, R)}\right\|_{\left(L^{p(\cdot)}\right)^{\prime}}}{|\Delta(t, R)|} \\
& \leq M_{2}(t) M_{2}^{\prime}(t) \frac{(x R)^{1 / p(t)} R^{1 / p^{\prime}(t)}}{|\Delta(t, R)|} \\
& \leq M_{2}(t) M_{2}^{\prime}(t) \frac{x^{1 / p(t)} R}{R / 2}=2 M_{2}(t) M_{2}^{\prime}(t) x^{1 / p(t)} .
\end{aligned}
$$

Combining (5.43), (5.45), and (4.3), we get for the same $x$ and $R$,

$$
\begin{aligned}
G_{1}(x R, R) & \geq M_{1}(t) M_{1}^{\prime}(t) \frac{(x R)^{1 / p(t)} R^{1 / p^{\prime}(t)}}{|\Delta(t, R)|} \\
& \geq M_{1}(t) M_{1}^{\prime}(t) \frac{x^{1 / p(t)} R}{C_{\Gamma, t} R}=\frac{M_{1}(t) M_{1}^{\prime}(t)}{C_{\Gamma, t}} x^{1 / p(t)} .
\end{aligned}
$$

From (5.47) and (5.48) it follows that

$$
\frac{M_{1}(t) M_{1}^{\prime}(t)}{C_{\Gamma, t}} x^{1 / p(t)} \leq\left(Q_{t}^{0} 1\right)(x) \leq 2 M_{2}(t) M_{2}^{\prime}(t) x^{1 / p(t)}, \quad x \in(0, \infty) .
$$

Since $1 \in A_{L^{p(\cdot)}}(\Gamma)$, the function $Q_{t}^{0} 1$ is regular and submultiplicative (see Lemmas 4.8 and 4.9). From the latter inequality it follows that

$$
\alpha\left(Q_{t}^{0} 1\right)=\beta\left(Q_{t}^{0} 1\right)=1 / p(t) .
$$

Combining the latter equalities with Lemma 4.8, we arrive at (5.40).

Theorem 5.9. Let $\Gamma$ be locally a Carleson curve at $t \in \Gamma$, let $w: \Gamma \rightarrow[0, \infty]$ be a weight, and let $p \in \mathcal{P}_{t}$. If $w \in A_{L^{p(\cdot)}}(\Gamma, t)$, then $\log w \in B M O(\Gamma, t)$ and

$$
\alpha\left(Q_{t} w\right)=1 / p(t)+\alpha\left(V_{t} w\right), \quad \beta\left(Q_{t} w\right)=1 / p(t)+\beta\left(V_{t} w\right) .
$$


Proof. Since $p \in \mathcal{P}_{t}$ and $\Gamma$ is locally a Carleson curve at $t$, in view of Lemma 5.8. $1 \in A_{L^{p(\cdot)}}(\Gamma, t)$. By Lemma 3.2 a), $\log w \in B M O(\Gamma, t)$. From Theorem [5.5] and (5.40) we get

$$
\begin{aligned}
1 / p(t)+\alpha\left(V_{t} w\right) \leq \alpha\left(Q_{t} w\right) & \leq \min \left\{1 / p(t)+\alpha\left(V_{t} w\right), 1 / p(t)+\beta\left(V_{t} w\right)\right\} \\
& =1 / p(t)+\alpha\left(V_{t} w\right), \\
1 / p(t)+\beta\left(V_{t} w\right) \geq \beta\left(Q_{t} w\right) & \geq \max \left\{1 / p(t)+\alpha\left(V_{t} w\right), 1 / p(t)+\beta\left(V_{t} w\right)\right\} \\
& =1 / p(t)+\beta\left(V_{t} w\right),
\end{aligned}
$$

that is, equalities (5.49) hold.

Lemma 5.10. Let $\Gamma$ be a Carleson curve, let $w: \Gamma \rightarrow[0, \infty]$ be a weight, and let $p \in \mathcal{P}$. If $w \in A_{L^{p(\cdot)}}(\Gamma)$, then $\log w \in B M O(\Gamma)$.

Proof. By analogy with Lemma 5.7 one can show that there exist constants $C>0$ and $M, M^{\prime} \in(0, \infty)$ such that

$$
\left\|\chi_{\Gamma(t, R)}\right\|_{L^{p(\cdot)}} \leq M R^{1 / p(t)}, \quad\left\|\chi_{\Gamma(t, R)}\right\|_{L^{p^{\prime}(\cdot)}} \leq M^{\prime} R^{1 / p^{\prime}(t)}
$$

for all $R \in(0, C)$ and all $t \in \Gamma$. Taking into account Lemma 2.4 as in Lemma 5.8 from the latter inequalities we obtain $1 \in A_{L^{p(\cdot)}}(\Gamma)$. Therefore, $\log w \in B M O(\Gamma)$, due to Lemma 3.2(b).

5.3. Indicator functions. In this subsection we generalize the notion of indicator functions (see [3, Ch. 3] and also [24, Section 7.2], 25, Section 2.5], [26, Section 3.3]) to the case of weighted Banach function spaces.

Suppose $\Gamma$ is a rectifiable Jordan curve, $w: \Gamma \rightarrow[0, \infty]$ is a weight, $X$ is a Banach function space.

Lemma 5.11. Let $\Gamma$ be locally a Carleson curve at $t \in \Gamma$. For every $x \in \mathbb{R}$, the function $W_{t} \eta_{t}^{x}$ is regular, submultiplicative, and

$$
\begin{aligned}
\alpha_{t}^{0}(x) & :=\alpha\left(W_{t} \eta_{t}^{x}\right)=\min \left\{\delta_{t}^{-} x, \delta_{t}^{+} x\right\}, \\
\beta_{t}^{0}(x) & :=\beta\left(W_{t} \eta_{t}^{x}\right)=\max \left\{\delta_{t}^{-} x, \delta_{t}^{+} x\right\} .
\end{aligned}
$$

This statement follows from local analogs of [3] Lemmas 1.15, 1.16, and Proposition 3.1].

For a complex number $\gamma \in \mathbb{C}$, we define a continuous function $\varphi_{t, \gamma}$ on $\Gamma \backslash\{t\}$ by

$$
\varphi_{t, \gamma}(\tau):=\left|(\tau-t)^{\gamma}\right|=|\tau-t|^{\operatorname{Re} \gamma} e^{-\operatorname{Im} \gamma \arg (\tau-t)}=|\tau-t|^{\operatorname{Re} \gamma}\left(\eta_{t}(\tau)\right)^{\operatorname{Im} \gamma} .
$$

Lemma 5.12. If $w \in A_{X}(\Gamma, t)$, then for every $\gamma \in \mathbb{C}$, the function $Q_{t}\left(\varphi_{t, \gamma} w\right)$ is regular, submultiplicative, and

$$
\begin{aligned}
\alpha\left(Q_{t}\left(\varphi_{t, \gamma} w\right)\right) & =\operatorname{Re} \gamma+\alpha\left(Q_{t}\left(\eta_{t}^{\operatorname{Im} \gamma} w\right)\right), \\
\beta\left(Q_{t}\left(\varphi_{t, \gamma} w\right)\right) & =\operatorname{Re} \gamma+\beta\left(Q_{t}\left(\eta_{t}^{\operatorname{Im} \gamma} w\right)\right) .
\end{aligned}
$$

Proof. This statement is proved similarly to [24, Lemma 7.2]. By a local analog of [3. Proposition 3.1], the function $W_{t} \varphi_{t, \operatorname{Re} \gamma}$ is regular and submultiplicative for every $\gamma \in \mathbb{C}$ and

$$
\alpha\left(W_{t} \varphi_{t, \operatorname{Re} \gamma}\right)=\beta\left(W_{t} \varphi_{t, \operatorname{Re} \gamma}\right)=\operatorname{Re} \gamma
$$


On the other hand, by Lemmas 4.8 4.9 the function $Q_{t} w$ is regular and submultiplicative. Then, by Theorem [5.2 the function $Q_{t}\left(\varphi_{t, \gamma} w\right)$ is regular and submultiplicative for every $\gamma \in \mathbb{C}$. In particular, the function $Q_{t}\left(\eta_{t}^{\operatorname{Im} \gamma} w\right)$ is regular and submultiplicative for every $\gamma \in \mathbb{C}$. From Theorem 5.2 and (5.53) it follows that

$$
\begin{aligned}
\alpha\left(Q_{t}\left(\eta_{t}^{\operatorname{Im} \gamma} w\right)\right)+\operatorname{Re} \gamma & \leq \alpha\left(Q_{t}\left(\varphi_{t, \gamma} w\right)\right) \\
& \leq \min \left\{\alpha\left(Q_{t}\left(\eta_{t}^{\operatorname{Im} \gamma} w\right)\right)+\operatorname{Re} \gamma, \beta\left(Q_{t}\left(\eta_{t}^{\operatorname{Im} \gamma} w\right)\right)+\operatorname{Re} \gamma\right\} \\
\beta\left(Q_{t}\left(\eta_{t}^{\operatorname{Im} \gamma} w\right)\right)+\operatorname{Re} \gamma & \geq \beta\left(Q_{t}\left(\varphi_{t, \gamma} w\right)\right) \\
& \geq \max \left\{\alpha\left(Q_{t}\left(\eta_{t}^{\operatorname{Im} \gamma} w\right)\right)+\operatorname{Re} \gamma, \beta\left(Q_{t}\left(\eta_{t}^{\operatorname{Im} \gamma} w\right)\right)+\operatorname{Re} \gamma\right\}
\end{aligned}
$$

From the latter inequalities we immediately obtain (5.51)-(5.52).

Lemma 5.13. If $w \in A_{X}(\Gamma, t)$ and $1 \in A_{X}(\Gamma, t)$, then for every $\gamma \in \mathbb{C}$, the function $V_{t}\left(\varphi_{t, \gamma} w\right)$ is regular, submultiplicative, and

$$
\begin{aligned}
\alpha\left(V_{t}\left(\varphi_{t, \gamma} w\right)\right) & =\operatorname{Re} \gamma+\alpha\left(V_{t}\left(\eta_{t}^{\operatorname{Im} \gamma} w\right)\right), \\
\beta\left(V_{t}\left(\varphi_{t, \gamma} w\right)\right) & =\operatorname{Re} \gamma+\beta\left(V_{t}\left(\eta_{t}^{\operatorname{Im} \gamma} w\right)\right) .
\end{aligned}
$$

Proof. By Lemma 3.2 (a), $\log w \in B M O(\Gamma, t)$. Then by Lemma 4.6] the function $V_{t} w$ is regular. The rest is proved by analogy with Lemma 5.12 with the help of Theorem 5.1

If $w \in A_{X}(\Gamma, t)$, then for every $x \in \mathbb{R}$, the function $Q_{t}\left(\eta_{t}^{x} w\right)$ is regular and submultiplicative, in view of Lemma 5.12. From Theorem 4.1 and Lemma 4.9 we deduce that the following functions are well defined for $x \in \mathbb{R}$ :

$$
\alpha_{t}^{*}(x):=\alpha\left(Q_{t}\left(\eta_{t}^{x} w\right)\right)=\alpha\left(Q_{t}^{0}\left(\eta_{t}^{x} w\right)\right), \quad \beta_{t}^{*}(x):=\beta\left(Q_{t}\left(\eta_{t}^{x} w\right)\right)=\beta\left(Q_{t}^{0}\left(\eta_{t}^{x} w\right)\right) .
$$

If, in addition, $1 \in A_{X}(\Gamma, t)$, then the function $V_{t}\left(\eta_{t}^{x} w\right)$ is regular and submultiplicative for each $x \in \mathbb{R}$, due to Lemma 5.13 Then Theorem 4.1 and Lemma 4.5 imply that the functions

$$
\alpha_{t}(x):=\alpha\left(V_{t}\left(\eta_{t}^{x} w\right)\right)=\alpha\left(V_{t}^{0}\left(\eta_{t}^{x} w\right)\right), \quad \beta_{t}(x):=\beta\left(V_{t}\left(\eta_{t}^{x} w\right)\right)=\beta\left(V_{t}^{0}\left(\eta_{t}^{x} w\right)\right)
$$

are well defined for all $x \in \mathbb{R}$.

The functions $\alpha_{t}^{*}, \beta_{t}^{*}$ are called the indicator functions of the triple $(\Gamma, X, w)$ at $t \in \Gamma$. The functions $\alpha_{t}, \beta_{t}$ are referred to as the indicator functions of the pair $(\Gamma, w)$ at $t \in \Gamma$. The functions $\alpha_{t}^{*}, \beta_{t}^{*}$ were introduced in 25] (see also [24, 26]) for rearrangement-invariant Banach function spaces. The functions $\alpha_{t}, \beta_{t}$ were defined in [3, Ch. 3] in the context of Lebesgue spaces and Muckenhoupt weights.

Lemma 5.14. The functions $\alpha_{t}, \alpha_{t}^{*}$ are concave, the functions $\beta_{t}, \beta_{t}^{*}$ are convex. In particular, $\alpha_{t}, \alpha_{t}^{*}$ and $\beta_{t}, \beta_{t}^{*}$ are continuous on $\mathbb{R}$.

Proof. By [35. Section 2.2, Property 6],

$$
\left\||f|^{\theta}|g|^{1-\theta}\right\|_{X} \leq\|f\|_{X}^{\theta}\|g\|_{X}^{1-\theta}, \quad \theta \in[0,1]
$$

for every $f, g \in X$. With the help of this property, one can prove concavity of $\alpha_{t}^{*}$ and convexity of $\beta_{t}^{*}$ similarly to [3, Proposition 3.20]. Concavity of $\alpha_{t}$ and convexity of $\beta_{t}$ are already proved there.

The following statement generalizes [26, Lemma 3.5]. 
Lemma 5.15. (a) If $w \in A_{X}(\Gamma, t)$, then for $x, y \in \mathbb{R}$,

$$
\begin{aligned}
& \alpha_{t}^{*}(x)+\alpha_{t}^{0}(y) \leq \alpha_{t}^{*}(x+y) \leq \min \left\{\alpha_{t}^{*}(x)+\beta_{t}^{0}(y), \beta_{t}^{*}(x)+\alpha_{t}^{0}(y)\right\}, \\
& \beta_{t}^{*}(x)+\beta_{t}^{0}(y) \geq \beta_{t}^{*}(x+y) \geq \max \left\{\alpha_{t}^{*}(x)+\beta_{t}^{0}(y), \beta_{t}^{*}(x)+\alpha_{t}^{0}(y)\right\} .
\end{aligned}
$$

(b) If $w \in A_{X}(\Gamma, t)$ and $1 \in A_{X}(\Gamma, t)$, then for $x, y \in \mathbb{R}$,

$$
\begin{aligned}
& \alpha_{t}(x)+\alpha_{t}^{0}(y) \leq \alpha_{t}(x+y) \leq \min \left\{\alpha_{t}(x)+\beta_{t}^{0}(y), \beta_{t}(x)+\alpha_{t}^{0}(y)\right\}, \\
& \beta_{t}(x)+\beta_{t}^{0}(y) \geq \beta_{t}(x+y) \geq \max \left\{\alpha_{t}(x)+\beta_{t}^{0}(y), \beta_{t}(x)+\alpha_{t}^{0}(y)\right\} .
\end{aligned}
$$

Proof. (a) From Lemmas 5.11 and 5.12 it follows that the functions $\alpha_{t}^{*}, \beta_{t}^{*}$ and $\alpha_{t}^{0}, \beta_{t}^{0}$ are well defined. Applying Theorem 5.2 to the weights $w:=\eta_{t}^{x} w$ and $\psi:=\eta_{t}^{y}$, we get Part (a). Part (b) is proved analogously with the help of Theorem [5.1] and Lemma 5.13

Corollary 5.16. Let $\Gamma$ be locally a Carleson curve at $t \in \Gamma$ such that $\delta_{t}^{-}=\delta_{t}^{+}=: \delta_{t}$. (a) If $w \in A_{X}(\Gamma, t)$, then

$$
\alpha_{t}^{*}(x)=\alpha\left(Q_{t} w\right)+\delta_{t} x, \quad \beta_{t}^{*}(x)=\beta\left(Q_{t} w\right)+\delta_{t} x \quad(x \in \mathbb{R}) .
$$

(b) If $w \in A_{X}(\Gamma, t)$ and $1 \in A_{X}(\Gamma, t)$, then

$$
\alpha_{t}(x)=\mu_{t}+\delta_{t} x, \quad \beta_{t}(x)=\nu_{t}+\delta_{t} x \quad(x \in \mathbb{R}) .
$$

Proof. (a) Since $\delta_{t}^{-}=\delta_{t}^{+}=\delta_{t}$, we have $\alpha_{t}^{0}(x)=\beta_{t}^{0}(x)=\delta_{t} x$. In that case from Lemma [5.15(a) we deduce that

$$
\alpha_{t}^{*}(y)+\delta_{t} x=\alpha_{t}^{*}(x+y), \quad \beta_{t}^{*}(y)+\delta_{t} x=\beta_{t}^{*}(x+y)
$$

for every $x, y \in \mathbb{R}$. Setting $y=0$ in (5.58), we arrive at (5.56). Part (b) is proved similarly.

5.4. Indicator functions for Nakano spaces. Let $\Gamma$ be a rectifiable Jordan curve, let $L^{p(\cdot)}$ be a Nakano space. Fix $t \in \Gamma$. For a weight $w \in A_{L^{p(\cdot)}}(\Gamma, t)$, put

$$
N_{t}:=\left\{\gamma \in \mathbb{C}: \quad \varphi_{t, \gamma} w \in A_{L^{p(\cdot)}}(\Gamma, t)\right\} .
$$

Lemma 5.17. Let $\Gamma$ be locally a Carleson curve at $t \in \Gamma$, let $p \in \mathcal{P}_{t}$, and let $w \in A_{L^{p(\cdot)}}(\Gamma, t)$. Then for every $\gamma \in N_{t}$,

$$
\alpha_{t}^{*}(\operatorname{Im} \gamma)=1 / p(t)+\alpha_{t}(\operatorname{Im} \gamma), \quad \beta_{t}^{*}(\operatorname{Im} \gamma)=1 / p(t)+\beta_{t}(\operatorname{Im} \gamma) .
$$

Proof. Let $\gamma \in N_{t}$. By Theorem 5.9

$$
\begin{aligned}
\alpha\left(Q_{t}\left(\varphi_{t, \gamma} w\right)\right) & =1 / p(t)+\alpha\left(V_{t}\left(\varphi_{t, \gamma} w\right)\right), \\
\beta\left(Q_{t}\left(\varphi_{t, \gamma} w\right)\right) & =1 / p(t)+\beta\left(V_{t}\left(\varphi_{t, \gamma} w\right)\right) .
\end{aligned}
$$

Note that by Lemma 5.8 $1 \in A_{L^{p(\cdot)}}(\Gamma, t)$. Therefore, we can apply Lemma 5.13 From (5.60)-(5.61), (5.51)-(5.52), and (5.54)-(5.55) it follows that

$\alpha\left(Q_{t}\left(\eta_{t}^{\operatorname{Im} \gamma} w\right)\right)=1 / p(t)+\alpha\left(V_{t}\left(\eta_{t}^{\operatorname{Im} \gamma} w\right)\right), \quad \beta\left(Q_{t}\left(\eta_{t}^{\operatorname{Im} \gamma} w\right)\right)=1 / p(t)+\beta\left(V_{t}\left(\eta_{t}^{\operatorname{Im} \gamma} w\right)\right)$, that is, equalities (5.59) hold.

Lemma 5.18. Let $\Gamma$ be locally a Carleson curve at $t \in \Gamma$ such that $\delta_{t}^{-}=\delta_{t}^{+}=0$, let $p \in \mathcal{P}_{t}$, and let $w \in A_{L^{p(\cdot)}}(\Gamma, t)$. Then for every every $x \in \mathbb{R}$,

$$
\alpha_{t}(x)=\mu_{t}, \quad \beta_{t}(x)=\nu_{t}, \quad \alpha_{t}^{*}(x)=1 / p(t)+\mu_{t}, \quad \beta_{t}^{*}(x)=1 / p(t)+\nu_{t},
$$

where $\mu_{t}, \nu_{t}$ are the indices of powerlikeness of the weight $w$ at $t$ defined by (4.1). 
Proof. By Lemma 5.8 $1 \in A_{L^{p(\cdot)}}(\Gamma, t)$. From Corollary[5.16] we get for every $x \in \mathbb{R}$,

$$
\alpha_{t}^{*}(x)=\alpha\left(Q_{t} w\right), \quad \beta_{t}^{*}(x)=\beta_{t}\left(Q_{t} w\right), \quad \alpha_{t}(x)=\mu_{t}, \quad \beta_{t}(x)=\nu_{t} .
$$

On the other hand, by Theorem 5.9

$$
\alpha\left(Q_{t} w\right)=1 / p(t)+\mu_{t}, \quad \beta\left(Q_{t} w\right)=1 / p(t)+\nu_{t} .
$$

Combining (5.63) and (5.64), we arrive at (5.62).

\section{FREDHOLM THEORY FOR SINGULAR INTEGRAL OPERATORS WITH BOUNDED MEASURABLE COEFFICIENTS}

6.1. The Cauchy singular integral operator. Let $\Gamma$ be a rectifiable Jordan curve. We provide $\Gamma$ with the counter-clockwise orientation. The curve $\Gamma$ divides the complex plane $\mathbb{C}$ into a bounded connected component $D^{+}$and an unbounded connected component $D^{-}$. Without loss of generality we suppose that $0 \in D^{+}$. Let $X$ be a Banach function space and $w: \Gamma \rightarrow[0, \infty]$ be a weight. Then the weighted Banach function space $X_{w}$ is a linear normed space which becomes a Banach function space whenever $w \in X$ and $1 / w \in X^{\prime}$ (see Lemma 2.5).

Theorem 6.1. Let $\Gamma$ be a rectifiable Jordan curve, let $w: \Gamma \rightarrow[0, \infty]$ be a weight, and let $X$ be a Banach function space. If the Cauchy singular integral operator $S$ is bounded on the weighted Banach function space $X_{w}$, then $w \in A_{X}(\Gamma)$.

This theorem was proved for weighted rearrangement-invariant Banach function spaces in a slightly different form in [24. Theorem 3.2] (see also 22, Theorem 4.3] and [3. Theorem 4.8]). First, as in [24, Lemma 3.3], by using the Landau lemma for the Banach function space $X$ (see [1, Ch. 1, Lemma 2.7]), we show that $w \in X$ and $1 / w \in X^{\prime}$. Then, by Lemma 2.5(b), the weighted Banach function space $X_{w}$ is itself a Banach function space. The proof of [24, Theorem 3.2] (see also [23, Section 3]) does not use the rearrangement-invariant property of the space $X$, so it works for arbitrary weighted Banach function spaces.

The question about the sufficiency of the condition $w \in A_{X}(\Gamma)$ for the boundedness of the Cauchy singular integral operator $S$ on weighted Banach function spaces $X_{w}$ is open. We know only that this condition is sufficient for the boundedness in the case of Lebesgue spaces $X=L^{p}, 1<p<\infty$, that is, when $A_{X}(\Gamma)=A_{p}(\Gamma)$ is the Muckenhoupt class (see, e.g., [3] Theorem 4.15]).

However, criteria for the boundedness of $S$ on Nakano spaces with Khvedelidze weights $L_{\varrho}^{p(\cdot)}$ were recently proved by V. M. Kokilashvili and S. G. Samko 30 under the condition that the contour $\Gamma$ is sufficiently nice.

Theorem 6.2. (see [30, Theorem 2]). Let $\Gamma$ be either a Lyapunov Jordan curve or a Radon Jordan curve without cusps, let $\varrho$ be a Khvedelidze weight (1.2), and let $p \in \mathcal{P}$. The Cauchy singular integral operator $S$ is bounded on the weighted Nakano space $L_{\varrho}^{p(\cdot)}$ if and only if

$$
0<\frac{1}{p\left(\tau_{k}\right)}+\lambda_{k}<1 \quad \text { for all } k \in\{1, \ldots, n\} .
$$

For weighted Lebesgue spaces $L_{\varrho}^{p}$ this result is classic, for Lyapunov curves it was proved by B. V. Khvedelidze [27] and for Radon curves without cusps by I. I. Danilyuk and V. Yu. Shelepov [9, Theorem 2]. The proofs and history can be found in [8, 16, 28, 40]. 
6.2. Singular integral operators. In the following we will assume that $\Gamma$ is a rectifiable Jordan curve, $X$ is a Banach function space, $w: \Gamma \rightarrow[0, \infty]$ is a weight such that

(B) the Cauchy singular integral operator $S$ is bounded on the weighted Banach function space $X_{w}$;

(R) the weighted Banach function space $X_{w}$ is reflexive.

Axiom (B) guarantees that, by Theorem 6.1 $w \in A_{X}(\Gamma)$. Therefore, $w \in X$ and $1 / w \in X^{\prime}$. Hence, $X_{w}$ is a Banach function space with the associate space $X_{1 / w}^{\prime}$ and

$$
L^{\infty} \subset X_{w} \subset L^{1} .
$$

On the other hand, if $w \in A_{X}(\Gamma)$, then $\Gamma$ is a Carleson curve. Axiom (R) implies that the Banach dual $\left(X_{w}\right)^{*}$ of $X_{w}$ coincides with its associate space $X_{1 / w}^{\prime}$ and the set $\mathcal{R}$ of all rational functions without poles on $\Gamma$ is dense in both $X_{w}$ and $X_{1 / w}^{\prime}$ (for details, see Subsection 2.4).

The above mentioned properties of weighted Banach functions spaces satisfying axioms (B) and (R) allow us to prove the following statements as in the case of weighted Lebsegue spaces (see, e.g., [16] Ch. 1] and [3] Ch. 6]). Detailed proofs can be found in [23, Ch. 2] (see also [24, 25]) for weighted rearrangement-invariant Banach function spaces $X_{w}$. Note that the assumption that $X$ is rearrangementinvariant is not essential and can be omitted there.

We denote by $\mathcal{K}\left(X_{w}\right)$ the closed two-sided ideal of all compact operators on $X_{w}$ in the Banach algebra $\mathcal{B}\left(X_{w}\right)$ of all bounded linear operators on $X_{w}$. As usual, $I$ is the identity operator on $X_{w}$ and $a I$ denotes the operator of multiplication by a measurable function $a: \Gamma \rightarrow \mathbb{C}$.

Lemma 6.3. If $a \in L^{\infty}$, then $a I \in \mathcal{B}\left(X_{w}\right)$ and $\|a I\|_{\mathcal{B}\left(X_{w}\right)} \leq\|a\|_{\infty}$.

Lemma 6.4. The operators

$$
P_{+}:=(I+S) / 2, \quad P_{-}:=(I-S) / 2
$$

are bounded projections on both $X_{w}$ and $X_{1 / w}^{\prime}$.

Lemma 6.5. If $a \in C$, then $a S-S a I \in \mathcal{K}\left(X_{w}\right)$.

On the weighted Banach function space $X_{w}$ (or on its dual $\left(X_{w}\right)^{*}=X_{1 / w}^{\prime}$ ) define the operator $H_{\Gamma}$ by $\left(H_{\Gamma} \varphi\right)(\tau):=e^{-i \theta_{\Gamma}(\tau)} \overline{\varphi(\tau)}$. Note that the operator $H_{\Gamma}$ is additive but $H_{\Gamma}(\alpha \varphi)=\bar{\alpha} \cdot H_{\Gamma} \varphi$ for $\alpha \in \mathbb{C}$. Evidently, $H_{\Gamma}^{2}=I$.

Lemma 6.6. The adjoint of $S \in \mathcal{B}\left(X_{w}\right)$ is $S^{*}=-H_{\Gamma} S H_{\Gamma} \in \mathcal{B}\left(X_{1 / w}^{\prime}\right)$.

For $a \in L^{\infty}$, put

$$
T_{a}:=P_{+} a P_{+}+P_{-}, \quad R_{a}:=a P_{+}+P_{-} .
$$

Lemma 6.7. Let $a \in L^{\infty}$. If one of the operators $T_{a}, R_{a}$ is semi-Fredholm, Fredholm, left-invertible, right-invertible, invertible, then the second operator has the same property. If the operators $T_{a}$ and $R_{a}$ are semi-Fredholm, then

$$
n\left(T_{a}\right)=n\left(R_{a}\right), \quad d\left(T_{a}\right)=d\left(R_{a}\right)
$$

Proof. By Lemmas 6.3 6.4 the operators $a I$ and $P_{ \pm}$are bounded on $X_{w}$. The rest follows from 21, Lemma 1.21]. 
So, it is sufficient to study only one of the operators $T_{a}, R_{a}$. We will formulate our main results for the operator $R_{a}$. This operator is usually called a singular integral operator with the coefficient $a$. It is well known that Fredholm properties of this operator are closely connected with the solvability of the Riemann-Hilbert boundary value problem (see, e.g., [6, 16, 38, ).

6.3. Hardy type subspaces. In view of Lemma 6.4 one can define the following subspaces of $X_{w}$ :

$$
\left(X_{w}\right)_{+}:=P_{+} X_{w}, \quad\left(X_{w}\right)_{-}^{0}:=P_{-} X_{w}, \quad\left(X_{w}\right)_{-}:=\left(X_{w}\right)_{-}^{0} \dot{+} \mathbb{C}
$$

the corresponding subspaces $\left(X_{1 / w}^{\prime}\right)_{+},\left(X_{1 / w}^{\prime}\right)_{-}^{0},\left(X_{1 / w}^{\prime}\right)_{-}$of $X_{1 / w}^{\prime}$ are defined analogously. Also put

$$
\begin{aligned}
L_{+}^{1} & :=\left\{f \in L^{1}: \int_{\Gamma} f(\tau) \tau^{n} d \tau=0 \quad \text { for } \quad n \geq 0\right\}, \\
\left(L^{1}\right)_{-}^{0} & :=\left\{f \in L^{1}: \int_{\Gamma} f(\tau) \tau^{n} d \tau=0 \text { for } n<0\right\}, \\
L_{-}^{1} & :=\left(L^{1}\right)_{-}^{0} \dot{+} \mathbb{C} .
\end{aligned}
$$

Lemma 6.8. (see [47 pp. 202-206]). We have $L_{+}^{1} \cap\left(L^{1}\right)_{-}^{0}=\{0\}$ and $L_{+}^{1} \cap L_{-}^{1}=\mathbb{C}$.

Lemma 6.9. (a) If $f \in\left(X_{w}\right)_{ \pm}$and $g \in\left(X_{1 / w}^{\prime}\right)_{ \pm}$, then $f g \in L_{ \pm}^{1}$. If, in addition, $f \in\left(X_{w}\right)_{-}^{0}$ or $g \in\left(X_{1 / w}^{\prime}\right)_{-}^{0}$, then $f g \in\left(L^{1}\right)_{-}^{0}$.

(b) We have,

$$
\left(X_{w}\right)_{+}=L_{+}^{1} \cap X_{w}, \quad\left(X_{w}\right)_{-}^{0}=\left(L^{1}\right)_{-}^{0} \cap X_{w}, \quad\left(X_{w}\right)_{-}=L_{-}^{1} \cap X_{w} .
$$

This lemma is proved by analogy with [3. Corollary 6.8] and [3, Lemma 6.11]. Here we essentially use Cauchy's theorem, Hölder's inequality for the weighted Banach function space $X_{w}$, and the density of $\mathcal{R}$ in $X_{w}$ and in $X_{1 / w}^{\prime}$ (see Corollary 2.11).

Lemma 6.10. Suppose $f_{ \pm}$is analytic in $D^{ \pm}$and continuous on $D^{ \pm} \cup \Gamma$ with the possible exception of finitely many points $t_{1}, \ldots, t_{m} \in \Gamma$. Suppose that $f_{ \pm} \mid \Gamma \in X_{w}$ and that $f_{ \pm}$admits the estimate

$$
\left|f_{ \pm}(z)\right| \leq M\left|z-t_{k}\right|^{-\mu} \quad(k=1 \ldots, m)
$$

with some $M>0, \mu>0$ for all $z \in D^{ \pm}$sufficiently close to $t_{k}$. Then $f_{ \pm} \in\left(X_{w}\right)_{ \pm}$.

This result goes back to S. Grudsky [17, Proposition 1.5] for Lebesgue spaces. To prove this statement, we should repeat the proof of [3, Lemma 6.10], replacing $L^{p}(\Gamma, w)$ by $X_{w}$ and using Lemma [6.9. For $\mu \in(0,1]$ and Lebesgue spaces this result was known for a long time [16, Ch. 2, Theorem 4.8]. We remark that for our purposes (see Lemma 7.1) we really need this analog of Grudsky's lemma allowing also the case $\mu>1$.

6.4. Two basic theorems. Let $G L^{\infty}$ denote the set of all functions in $L^{\infty}$ which are invertible in $L^{\infty}$, that is, the set of functions $a \in L^{\infty}$ such that

$$
\underset{\tau \in \Gamma}{\operatorname{ess} \inf }|a(\tau)|>0 .
$$

Theorem 6.11. Let $a, b \in L^{\infty}$. If the operator $a P_{+}+b P_{-}$is semi-Fredholm in $X_{w}$, then $a, b \in G L^{\infty}$. 
Theorem 6.12. If $a \in G L^{\infty}$, then $\min \left\{n\left(R_{a}\right), d\left(R_{a}\right)\right\}=0$.

Theorem 6.12 was proved by L. Coburn 7 for Toeplitz operators on $L^{2}(\mathbb{T})$. In the form presented here Theorems 6.11 and 6.12 were proved by I. B. Simonenko in 51] for Lebesgue spaces with Khvedelidze weights over Lyapunov curves. For a detailed discussion of these theorems for weighted Lebesgue spaces, see [3] Section 6.6] and 16. Sections 7.4 and 7.5]. In our case the proofs are developed analogously on the basis of the results of Subsections 6.2 6.3 and the Lusin-Privalov theorem (see, e.g., [47, p. 292]).

6.5. The local principle of Simonenko type. Two functions $a, b \in L^{\infty}$ are said to be locally equivalent at a point $t \in \Gamma$ if

$$
\inf \left\{\|(a-b) c\|_{\infty}: \quad c \in C, \quad c(t)=1\right\}=0 .
$$

Theorem 6.13. Let $a \in L^{\infty}$. Suppose for each $t \in \Gamma$ we are given a function $a_{t} \in L^{\infty}$ which is locally equivalent to a at $t$. If the operators $R_{a_{t}}$ are Fredholm in $X_{w}$ for all $t \in \Gamma$, then $R_{a}$ is Fredholm in $X_{w}$.

For weighted Lebesgue spaces, this theorem is known as Simonenko's local principle 50. More information about localization techniques can be found, e.g., in 3] 5, 16, 36. Theorem 6.13 can be proved similarly to [3, Theorem 6.30] with the help of Lemmas 6.5 and 6.7.

6.6. Wiener-Hopf factorization. We say that a function $a \in L^{\infty}$ admits a Wiener-Hopf factorization in the weighted Banach function space $X_{w}$ if $1 / a \in L^{\infty}$ and $a$ can be written in the form

$$
a(t)=a_{-}(t) t^{\kappa} a_{+}(t) \quad \text { a.e. on } \Gamma,
$$

where $\kappa \in \mathbb{Z}$, and the factors $a_{ \pm}$enjoy the following properties:

(i) $a_{-} \in\left(X_{w}\right)_{-}, \quad 1 / a_{-} \in\left(X_{1 / w}^{\prime}\right)_{-}, \quad a_{+} \in\left(X_{1 / w}^{\prime}\right)_{+}, \quad 1 / a_{+} \in\left(X_{w}\right)_{+}$,

(ii) the operator $\left(1 / a_{+}\right) S a_{+} I$ is bounded on $X_{w}$.

One can prove that the number $\kappa$ is uniquely determined.

Theorem 6.14. A function $a \in L^{\infty}$ admits a Wiener-Hopf factorization (6.2) in the reflexive weighted Banach function space $X_{w}$ if and only if the operator $R_{a}$ is Fredholm in $X_{w}$. If $R_{a}$ is Fredholm, then its index is equal to $-\kappa$.

This theorem goes back to I. B. Simonenko 49 151. For more about this topic we refer to [3, Section 6.12], [5, Section 5.5], 16. Section 8.3] and also to [6, 38 in the case of weighted Lebesgue spaces. Simonenko's result was generalized by the author to the case of reflexive Orlicz spaces [22] Theorem 5.6] and to the case of reflexive rearrangement-invariant spaces [24, Theorem 6.10]. In the case of reflexive weighted Banach function spaces the proof is developed by analogy. The proof is essentially based on the density of $\mathcal{R}$ in $X_{w}$ and in $X_{1 / w}^{\prime}$, Lemmas 6.8 6.9 and Theorems 6.11 6.12 Detailed proofs for the results of this section can be found in [23. Ch. 2] for weighted Banach function spaces $X_{w}$ provided $X$ is rearrangementinvariant. Let us remind that this assumption can be simply omitted. 


\section{FREDHOLMNESS OF SINGULAR INTEGRAL OPERATORS IN WEIGHTED BANACH FUNCTION SPACES}

7.1. Local representatives. Fix $t \in \Gamma$. For a function $a \in P C \cap G L^{\infty}$ we construct a "canonical" function $g_{t, \gamma}$ which is locally equivalent to $a$ at the point $t \in \Gamma$. The interior and the exterior of the unit circle can be conformally mapped onto $D^{+}$ and $D^{-}$of $\Gamma$, respectively, so that the point 1 is mapped to $t$, and the points $0 \in D^{+}$and $\infty \in D^{-}$remain fixed. Let $\Lambda_{0}$ and $\Lambda_{\infty}$ denote the images of $[0,1]$ and $[1, \infty) \cup\{\infty\}$ under this map. The curve $\Lambda_{0} \cup \Lambda_{\infty}$ joins 0 to $\infty$ and meets $\Gamma$ at exactly one point, namely $t$. Let $\arg z$ be a continuous branch of argument in $\mathbb{C} \backslash\left(\Lambda_{0} \cup \Lambda_{\infty}\right)$. For $\gamma \in \mathbb{C}$, define the function $z^{\gamma}:=|z|^{\gamma} e^{i \gamma \arg z}$, where $z \in \mathbb{C} \backslash\left(\Lambda_{0} \cup \Lambda_{\infty}\right)$. Clearly, $z^{\gamma}$ is an analytic function in $\mathbb{C} \backslash\left(\Lambda_{0} \cup \Lambda_{\infty}\right)$. The restriction of $z^{\gamma}$ to $\Gamma \backslash\{t\}$ will be denoted by $g_{t, \gamma}$. Obviously, $g_{t, \gamma}$ is continuous and nonzero on $\Gamma \backslash\{t\}$.

Since $a(t \pm 0) \neq 0$, we can define $\gamma_{t}=\gamma \in \mathbb{C}$ by the formulas

$$
\operatorname{Re} \gamma_{t}:=\frac{1}{2 \pi} \arg \frac{a(t-0)}{a(t+0)}, \quad \operatorname{Im} \gamma_{t}:=-\frac{1}{2 \pi} \log \left|\frac{a(t-0)}{a(t+0)}\right|,
$$

where we can take any value of $\arg (a(t-0) / a(t+0))$, which implies that any two choices of $\operatorname{Re} \gamma_{t}$ differ by an integer only. Clearly, there is a constant $c_{t} \in \mathbb{C} \backslash\{0\}$ such that $a(t \pm 0)=c_{t} g_{t, \gamma_{t}}(t \pm 0)$, which means that $a$ is locally equivalent to $c_{t} g_{t, \gamma_{t}}$ at the point $t \in \Gamma$.

\subsection{Sufficient conditions for factorability of the local representative.}

Lemma 7.1. If, for some $k \in \mathbb{Z}$ and $\gamma \in \mathbb{C}$, the operator $\varphi_{t, k-\gamma} S \varphi_{t, \gamma-k} I$ is bounded on the weighted Banach function space $X_{w}$, then

$$
g_{t, \gamma}(\tau)=(1-t / \tau)^{k-\gamma} \tau^{k}(\tau-t)^{\gamma-k}, \quad \tau \in \Gamma \backslash\{t\}
$$

is a Wiener-Hopf factorization of the function $g_{t, \gamma}$ in $X_{w}$.

Proof. Since the operator $\varphi_{t, k-\gamma} S \varphi_{t, k-\gamma}^{-1} I$ is bounded on $X_{w}$, the operator $S$ is bounded on the weighted Banach function space $X_{\varphi_{t, k-\gamma} w}$. By Theorem 6.1, $\varphi_{t, k-\gamma} w \in A_{X}(\Gamma)$. In that case $\Gamma$ is a Carleson curve and $\varphi_{t, k-\gamma} w \in X$, whence $\varphi_{t, k-\gamma} \in X_{w}$.

Let us show that $(\tau-t)^{k-\gamma} \in\left(X_{w}\right)_{+}$. The function $f(z):=(z-t)^{k-\gamma}$ is analytic in $D^{+}$and continuous on $D^{+} \cup(\Gamma \backslash\{t\})$. For $z \in D^{+}$,

$$
|f(z)|=\left|(z-t)^{k-\gamma}\right|=|z-t|^{k-\operatorname{Re} \gamma-\Theta_{t}(z) \operatorname{Im} \gamma},
$$

where $\Theta_{\tau}(z):=\arg (z-t) /(-\log |z-t|)$. As in [24, Theorem 7.7] and [3, Lemma 7.1] with the help of Lemma 4.2 one can show that there is a constant $M_{t} \in(0, \infty)$ such that

$$
|f(z)| \leq|z-t|^{k-\operatorname{Re} \gamma} e^{M_{t}|\operatorname{Im} \gamma|(-\log |z-t|)}=|z-t|^{k-\operatorname{Re} \gamma-M_{t}|\operatorname{Im} \gamma|}
$$

for all $z$ in a small neighborhood of $t$. By Lemma 6.10 $(\tau-t)^{k-\gamma} \in\left(X_{w}\right)_{+}$. Analogously one can prove that

$$
(\tau-t)^{\gamma-k} \in\left(X_{1 / w}^{\prime}\right)_{+}, \quad(1-t / \tau)^{k-\gamma} \in\left(X_{w}\right)_{-}, \quad(1-t / \tau)^{\gamma-k} \in\left(X_{1 / w}^{\prime}\right)_{-} .
$$

These facts together with the boundedness of $\varphi_{t, k-\gamma} S \varphi_{t, \gamma-k} I$ on the space $X_{w}$ show that (7.2) is indeed a Wiener-Hopf factorization of the function $g_{t, \gamma}$. 


\subsection{Necessary conditions for factorability of the local representative.}

Theorem 7.2. If the function $g_{t, \gamma}$ admits a Wiener-Hopf factorization in the weighted Banach function space $X_{w}$, then

$$
-\operatorname{Re} \gamma+\theta \alpha_{t}^{*}(-\operatorname{Im} \gamma)+(1-\theta) \beta_{t}^{*}(-\operatorname{Im} \gamma) \notin \mathbb{Z}
$$

for all $\theta \in[0,1]$. Moreover, there exists an $l \in \mathbb{Z}$ such that $\varphi_{t, l-\gamma} w \in A_{X}(\Gamma)$.

Proof. The idea of the proof (in the case of weighted Lebesgue spaces) goes back to I. Spitkovsky [52] and it was further developed by A. Böttcher and Yu. I. Karlovich 3] Proposition 7.2]. This idea was applied to the proof in the case of reflexive rearrangement-invariant Banach function spaces (with weights) in [24, Theorem 7.6] and 25]. Theorem 4.1]. Since, for our (more general) case, the arguments are the same, we point out only the main steps.

By Theorem 6.14 the operator $g_{t, \gamma} P_{+}+P_{-}$is Fredholm. Then there exists a $c>0$ such that the operators $g_{t, \gamma-\varepsilon} P_{+}+P_{-}$are Fredholm for all $\varepsilon \in(-c, c)$. Applying Theorem 6.14 again, we infer that all functions $g_{t, \gamma-\varepsilon}$ admit a WienerHopf factorization in $X_{w}$. By using its definition, one can show that there exists an $l \in \mathbb{Z}$ such that the operators $\varphi_{t, l-\gamma+\varepsilon} S \varphi_{t, l-\gamma+\varepsilon}^{-1} I$ are bounded on $X_{w}$ for all $\varepsilon \in(-c, c)$. In that case, by Theorem 6.1] $\varphi_{t, l-\gamma+\varepsilon} w \in A_{X}(\Gamma) \subset A_{X}(\Gamma, t)$. By Lemma 4.9

$$
0 \leq\left(Q_{t}\left(\varphi_{t, l-\gamma+\varepsilon} w\right)\right) \leq \beta\left(Q_{t}\left(\varphi_{t, l-\gamma+\varepsilon} w\right)\right) \leq 1 .
$$

From Lemma 5.12 and (7.4) it follows that

$$
0 \leq l+\varepsilon-\operatorname{Re} \gamma+\alpha_{t}^{*}(-\operatorname{Im} \gamma) \leq l+\varepsilon-\operatorname{Re} \gamma+\beta_{t}^{*}(-\operatorname{Im} \gamma) \leq 1
$$

for all $\varepsilon \in(-c, c)$. Hence,

$$
-l<-\operatorname{Re} \gamma+\theta \alpha_{t}^{*}(-\operatorname{Im} \gamma)+(1-\theta) \beta_{t}^{*}(-\operatorname{Im} \gamma)<l-1
$$

for every $\theta \in[0,1]$. Thus, (7.3) holds for every $\theta \in[0,1]$.

7.4. Necessary conditions for Fredholmness. Now we are in a position to state the main result of this paper.

Theorem 7.3. Let $\Gamma$ be a rectifiable Jordan curve, let $w: \Gamma \rightarrow[0, \infty]$ be a weight, and let $X$ be a Banach function space. Suppose the Cauchy singular integral operator $S$ is bounded on the weighted Banach function space $X_{w}$ and $X_{w}$ is reflexive. If the operator a $P_{+}+P_{-}$, where $a \in P C$, is Fredholm in $X_{w}$, then $a \in G L^{\infty}$ and

$$
\begin{aligned}
& -\quad \frac{1}{2 \pi} \arg \frac{a(t-0)}{a(t+0)} \\
& +\quad \theta \alpha_{t}^{*}\left(\frac{1}{2 \pi} \log \left|\frac{a(t-0)}{a(t+0)}\right|\right)+(1-\theta) \beta_{t}^{*}\left(\frac{1}{2 \pi} \log \left|\frac{a(t-0)}{a(t+0)}\right|\right) \notin \mathbb{Z}
\end{aligned}
$$

for all $t \in \Gamma$ and all $\theta \in[0,1]$.

Proof. The proof is developed by analogy with the proof of necessity part of [24, Theorem 7.8] (see also [3, Proposition 7.3]).

If $R_{a}$ is Fredholm, then, by Theorem 6.11 $a \in G L^{\infty}$. Fix an arbitrary $t \in \Gamma$. Choose $\gamma=\gamma_{t} \in \mathbb{C}$ as in (7.1). Then the function $a$ is locally equivalent to $c_{t} g_{t, \gamma_{t}}$ at the point $t$, where $c_{t} \in \mathbb{C} \backslash\{0\}$ is some constant. If $\tau \in \Gamma \backslash\{t\}$, then $g_{t, \gamma_{t}}$ is continuous and nonzero at $\tau$. Hence, it is locally equivalent to the nonzero constant $b_{\tau}:=g_{t, \gamma_{t}}(\tau)$ at $\tau$. Clearly, the operator $R_{b_{\tau}}:=b_{\tau} P_{+}+P_{-}$is invertible, 
$\left(b_{\tau} P_{+}+P_{-}\right)^{-1}=b_{\tau}^{-1} P_{+}+P_{-}$. Therefore, the operator $R_{b_{\tau}}$ is Fredholm for every $\tau \in \Gamma \backslash\{t\}$. Remind that the function $g_{t, \gamma_{t}}$ is locally equivalent to the function $c_{t}^{-1} a$. Since

$$
R_{c_{t}^{-1}} R_{a}=P_{+} c_{t}^{-1} a P_{+}+P_{-}=T_{c_{t}^{-1} a}
$$

and the operator $R_{c_{t}^{-1}}$ is invertible, from Lemma 6.7 and (7.6) it follows that $R_{a}$ is Fredholm if and only if $R_{c_{t}^{-1} a}$ is Fredholm. Therefore, applying Theorem 6.13 we infer that the operator $R_{g_{t, \gamma_{t}}}$ is Fredholm. By Theorem 6.14 the function $g_{t, \gamma_{t}}$ admits a Wiener-Hopf factorization in $X_{w}$. From Theorem 7.2 it follows that

$$
-\operatorname{Re} \gamma_{t}+\theta \alpha_{t}^{*}\left(-\operatorname{Im} \gamma_{t}\right)+(1-\theta) \beta_{t}^{*}\left(-\operatorname{Im} \gamma_{t}\right) \notin \mathbb{Z}
$$

for all $\theta \in[0,1]$. Since $t \in \Gamma$ is arbitrary, from (17.1) and (17.7) we conclude that (7.5) holds for every $t \in \Gamma$ and every $\theta \in[0,1]$.

7.5. Lower estimates for essential norms. For an operator $A \in \mathcal{B}\left(X_{w}\right)$, let

$$
|A|_{X_{w}}:=\inf _{K \in \mathcal{K}\left(X_{w}\right)}\|A+K\|_{\mathcal{B}\left(X_{w}\right)}
$$

be its essential norm in $X_{w}$.

Theorem 7.4. Let $\Gamma$ be a rectifiable Jordan curve, let $w: \Gamma \rightarrow[0, \infty]$ be a weight, and let $X$ be a Banach function space. If the Cauchy singular integral operator $S$ is bounded on the weighted Banach function space $X_{w}$ and $X_{w}$ is reflexive, then

$$
|S|_{X_{w}} \geq \cot \left(\pi \Lambda_{\Gamma, X, w} / 2\right), \quad\left|P_{ \pm}\right|_{X_{w}} \geq 1 / \sin \left(\pi \Lambda_{\Gamma, X, w}\right),
$$

where

$$
\Lambda_{\Gamma, X, w}:=\inf _{t \in \Gamma} \min \left\{\alpha\left(Q_{t} w\right), 1-\beta\left(Q_{t} w\right)\right\} .
$$

This statement is proved by a literal repetition of the proof of [25] Theorem 4.5] using the scheme of [16, Ch. 9, Theorem 9.1]. One can find more information about estimates of (essential) norms on weighted Lebesgue spaces in [16, Ch. 13] and 36. Ch. 2].

\section{Fredholmness of SingUlar INTEGRAL OPERATORS IN WEIGHTED NAKANO SPACES}

8.1. Necessary conditions for Fredholmness. The necessary conditions for the Fredholmness of $R_{a}$ in weighted Nakano spaces have a simpler form than in the general case because we can replace the indicator functions $\alpha_{t}^{*}$ and $\beta_{t}^{*}$ by the indicator functions $1 / p(t)+\alpha_{t}$ and $1 / p(t)+\beta_{t}$, respectively. More precisely, the next theorem is true.

Theorem 8.1. Let $\Gamma$ be a rectifiable Jordan curve, let $w: \Gamma \rightarrow[0, \infty]$ be a weight, and let $p \in \mathcal{P}$. Suppose the Cauchy singular integral operator is bounded on the weighted Nakano space $L_{w}^{p(\cdot)}$. If the operator $a P_{+}+P_{-}$, where $a \in P C$, is Fredholm in $L_{w}^{p(\cdot)}$, then $a \in G L^{\infty}$ and

$$
\begin{aligned}
& -\frac{1}{2 \pi} \arg \frac{a(t-0)}{a(t+0)}+\frac{1}{p(t)} \\
& +\theta \alpha_{t}\left(\frac{1}{2 \pi} \log \left|\frac{a(t-0)}{a(t+0)}\right|\right)+(1-\theta) \beta_{t}\left(\frac{1}{2 \pi} \log \left|\frac{a(t-0)}{a(t+0)}\right|\right) \notin \mathbb{Z}
\end{aligned}
$$

for all $t \in \Gamma$ and all $\theta \in[0,1]$. 
Proof. Since $p \in \mathcal{P}$, inequalities (5.25) are satisfied. In that case, by Lemma 2.4 the non-weighted Nakano spaces $L^{p(\cdot)}$ is reflexive. On the other hand, by Theorem 6.1. $w \in L^{p(\cdot)}$ and $1 / w \in\left(L^{p(\cdot)}\right)^{\prime}$. Then the weighted Nakano space $L_{w}^{p(\cdot)}$ is also reflexive, due to Corollary 2.8 Thus, all assumptions of Theorem 7.3 are satisfied and we can repeat its proof. In view of Theorem 7.2 there exists an $l \in \mathbb{Z}$ such that $\varphi_{t, l-\gamma_{t}} w \in A_{L^{p(\cdot)}}(\Gamma)$, where $\gamma_{t}$ is given by (7.1). In that case, by Lemma 5.17

$$
\begin{aligned}
& -\operatorname{Re} \gamma_{t}+\theta \alpha_{t}^{*}\left(-\operatorname{Im} \gamma_{t}\right)+(1-\theta) \beta_{t}^{*}\left(-\operatorname{Im} \gamma_{t}\right) \\
& =-\operatorname{Re} \gamma_{t}+1 / p(t)+\theta \alpha_{t}\left(-\operatorname{Im} \gamma_{t}\right)+(1-\theta) \beta_{t}\left(-\operatorname{Im} \gamma_{t}\right)
\end{aligned}
$$

Therefore, we can replace condition (7.5) by condition (8.1) in the case of weighted Nakano spaces.

For Lebesgue spaces $L_{w}^{p}$ with Muckenhoupt weights $w$ (that is, in the case when $p(\cdot)$ is constant), condition (8.1) becomes also sufficient for the Fredholmness of $R_{a}$ (see [3, Proposition 7.3]).

\subsection{Lower estimates for essential norms.}

Theorem 8.2. Let $\Gamma$ be a rectifiable Jordan curve, let $w: \Gamma \rightarrow[0, \infty]$ be a weight, and let $p \in \mathcal{P}$. If the Cauchy singular integral operator is bounded on the weighted Nakano space $L_{w}^{p(\cdot)}$, then

$$
|S|_{L_{w}^{p(\cdot)}} \geq \cot \left(\pi \Lambda_{\Gamma, p, w} / 2\right), \quad\left|P_{ \pm}\right|_{L_{w}^{p(\cdot)}} \geq 1 / \sin \left(\pi \Lambda_{\Gamma, p, w}\right)
$$

where

$$
\Lambda_{\Gamma, p, w}:=\inf _{t \in \Gamma} \min \left\{\frac{1}{p(t)}+\mu_{t}, 1-\frac{1}{p(t)}-\nu_{t}\right\} .
$$

By Theorem 6.1] $w \in A_{L^{p(\cdot)}}(\Gamma)$. Therefore, the latter theorem immediately follows from Theorem 7.4 and Theorem [5.9

If $\log w \in V M O(\Gamma)$ (in particular, if $w=1$ ), then from Lemma 4.7 and (5.25) it follows that

$$
\begin{aligned}
\Lambda_{\Gamma, p, w} & =\inf _{t \in \Gamma} \min \left\{\frac{1}{p(t)}, 1-\frac{1}{p(t)}\right\}=\min \left\{\inf _{t \in \Gamma} \frac{1}{p(t)}, 1-\sup _{t \in \Gamma} \frac{1}{p(t)}\right\} \\
& =\min \left\{1 / p^{*}, 1-1 / p_{*}\right\} .
\end{aligned}
$$

\subsection{Fredholm criterion.}

Theorem 8.3. Let $\Gamma$ be either a Lyapunov Jordan curve or a Radon Jordan curve without cusps, let $p \in \mathcal{P}$, and let $\varrho$ be a Khvedelidze weight (1.2) satisfying (6.1). Then the operator $a P_{+}+P_{-}$, where $a \in P C$, is Fredholm in the weighted Nakano space $L_{\varrho}^{p(\cdot)}$ if and only if

$$
a(t \pm 0) \neq 0, \quad-\frac{1}{2 \pi} \arg \frac{a(t-0)}{a(t+0)}+\frac{1}{p(t)}+\lambda(t) \notin \mathbb{Z}
$$

for all $t \in \Gamma$, where

$$
\lambda(t)= \begin{cases}\lambda_{k}, & \text { if } t=\tau_{k}, \quad k \in\{1, \ldots, n\}, \\ 0, & \text { if } t \notin \Gamma \backslash\left\{\tau_{1}, \ldots, \tau_{n}\right\} .\end{cases}
$$


Proof. By Theorem 6.2 the operator $S$ is bounded on the (reflexive) weighted Nakano space $L_{o}^{p(\cdot)}$.

Necessity. By Proposition 4.4 for Lyapunov curves and Radon curves without cusps, we have $\delta_{t}^{-}=\delta_{t}^{+}=0$ whenever $t \in \Gamma$. By Lemma 5.18 the indicator functions of the pair $(\Gamma, \varrho)$ are constants $\alpha_{t}(x)=\mu_{t}, \beta_{t}(x)=\nu_{t}$ for $x \in \mathbb{R}$, where the indices of powerlikeness $\mu_{t}, \nu_{t}$ of the Khvedelidze weight (1.2) coincide with $\lambda(t)$ given by (8.3). Thus,

$$
\theta \alpha_{t}\left(\frac{1}{2 \pi} \log \left|\frac{a(t-0)}{a(t+0)}\right|\right)+(1-\theta) \beta_{t}\left(\frac{1}{2 \pi} \log \left|\frac{a(t-0)}{a(t+0)}\right|\right)=\lambda(t)
$$

for every $\theta \in[0,1]$ and every $t \in \Gamma$. Therefore, the necessity of conditions (8.2) follows from Theorem 8.1. The necessity part is proved.

Sufficiency. From (8.2) it follows that for every $t \in \Gamma$, there exists an $m_{t} \in \mathbb{Z}$ such that

$$
0<m_{t}-\operatorname{Re} \gamma_{t}+\frac{1}{p(t)}+\lambda(t)<1,
$$

where $\gamma_{t}$ is given by (7.1). By Theorem 6.2 the operator $S$ is bounded on the weighted Nakano space $L_{\widetilde{\varrho}_{t}}^{p(\cdot)}$, where

$$
\widetilde{\varrho}_{t}(\tau):=|\tau-t|^{m_{t}-\operatorname{Re} \gamma_{t}} \varrho(\tau), \quad \tau \in \Gamma .
$$

In view of (5.50) and Proposition 4.4 there exist constants $C_{1}(t), C_{2}(t) \in(0, \infty)$ such that

$$
C_{1}(t) \widetilde{\varrho}_{t}(\tau) \leq \varphi_{t, m_{t}-\gamma_{t}}(\tau) \leq C_{2}(t) \widetilde{\varrho}_{t}(\tau), \quad \tau \in \Gamma \backslash\{t\} .
$$

Therefore, $S \in \mathcal{B}\left(L_{\widetilde{\varrho}_{t}}^{p(\cdot)}\right)$ if and only if $\varphi_{t, m_{t}-\gamma_{t}} S \varphi_{t, \gamma_{t}-m_{t}} I \in \mathcal{B}\left(L_{\varrho}^{p(\cdot)}\right)$. By Lemma[7.1] the function $g_{t, \gamma_{t}}$ admits a Wiener-Hopf factorization in the weighted Nakano space $L_{\varrho}^{p(\cdot)}$. Due to Theorem 6.14 for every $t \in \Gamma$, the operator $g_{t, \gamma_{t}} P_{+}+P_{-}$is Fredholm. Then the operator $c g_{t, \gamma_{t}} P_{+}+P_{-}$is Fredholm for $c \in \mathbb{C} \backslash\{0\}$ (see the proof of Theorem [7.3).

Since the function $c_{t} g_{t, \gamma_{t}}$ with a specially chosen constant $c_{t} \in \mathbb{C} \backslash\{0\}$ is locally equivalent to the function $a \in P C$ at every point $t \in \Gamma$, in view of Theorem 6.13. the operator $R_{a}=a P_{+}+P_{-}$is Fredholm in the weighted Nakano space $L_{\varrho}^{p(\cdot)}$.

In Theorem 8.3 the coefficient $a$ can have a countable set of jumps. If $a$ has only a finite number of jumps and $\varrho=1$, this result was obtained in [31, Theorem A] (as well as a formula for the index of the operator $R_{a}$ ). Note that the transition from finitely many to infinitely many jumps is more or less standard (see [16, Section 9.8] for Lebesgue spaces with Khvedelidze weights over Lyapunov curves), using the stability of Fredholm operators and localization techniques (see Section 6.5). We give the proof of Theorem 8.3 here for completeness. For Lebesgue spaces with Khvedelidze weights over Lyapunov curves the corresponding result was obtained in the late sixties by I. Gohberg and N. Krupnik [16, Ch. 9].

Acknowledgments. I would like to express my deep gratitude to Professor Lech Maligranda (Luleå University of Technology, Sweden) for historical remarks concerning Nakano spaces and to Professor Albrecht Böttcher (Chemnitz Technical University, Germany) for useful discussions. 


\section{REFERENCES}

[1] C. Bennett, R. Sharpley, Interpolation of Operators, Pure and Applied Mathematics, 129. Academic Press, Boston, 1988.

[2] E. I. Berezhnoi, Two-weighted estimations for the Hardy-Littlewood maximal function in ideal Banach spaces, Proc. Amer. Math. Soc., 127 (1999), 79-87.

[3] A. Böttcher, Yu. I. Karlovich, Carleson Curves, Muckenhoupt Weights, and Toeplitz Operators, Progress in Mathematics, 154. Birkhäuser Verlag, Basel, Boston, Berlin, 1997.

[4] A. Böttcher, Yu. I. Karlovich, Cauchy's singular integral operator and its beautiful spectrum. In: "Systems, approximation, singular integral operators, and related topics" (Bordeaux, 2000), pp. 109-142, Operator Theory: Advances and Applications, 129. Birkhäuser Verlag, Basel, 2001.

[5] A. Böttcher, B. Silbermann, Analysis of Toeplitz Operators, Springer-Verlag, Berlin, 1990.

[6] K. F. Clancey, I. Gohberg, Factorization of Matrix Functions and Singular Integral Operators, Operator Theory: Advances and Applications, 3. Birkhäuser Verlag, Basel, 1981.

[7] L. A. Coburn, Weyl's theorem for nonnormal operators, Michigan Math. J., 13 (1966), 285288.

[8] I. I. Danilyuk, Nonregular Boundary Value Problems in the Plane, Nauka, Moscow, 1975 (in Russian).

[9] I. I. Danilyuk, V. Yu. Shelepov, Boundedness in $L_{p}$ of a singular operator with Cauchy kernel along a curve of bounded rotation, Dokl. Akad. Nauk SSSR, 174 (1967), 514-517 (in Russian). English translation: Soviet Math. Dokl., 8 (1967), 654-657.

[10] L. Diening, Maximal functions on generalized Lebesgue spaces $L^{p(x)}$, Mathematische Fakultät, Albert-Ludvigs-Universität Freiburg (2002), Preprint Nr. 02/2002-16.01.2002.

[11] E. M. Dynkin, Methods of the theory of singular integrals (Hilbert transform and CalderónZygmund theory), Itogi nauki i tehniki VINITI, Ser. Sovrem. probl. mat., 15 (1987), $197-$ 292 (in Russian). English translation: Commutative harmonic analysis I. General survey. Classical aspects, Encycl. Math. Sci., 15 (1991), 167-259.

[12] D. E. Edmunds, J. Lang, A. Nekvinda, On $L^{p(x)}$ norms, R. Soc. Lond. Proc. Ser. A Math. Phys. Eng. Sci., 455 (1999), no. 1981, 219-225.

[13] R. J. Fleming, J. E. Jamison, A. Kamińska, Isometries of Musielak-Orlicz spaces. In: "Function spaces" (Edwardsville, IL, 1990), pp. 139-154. Lecture Notes in Pure and Appl. Math., 136. Dekker, New York, 1992.

[14] D. Gaier, Lectures on Complex Approximation, Birkhäuser Boston, Inc., Boston, MA, 1987.

[15] I. Genebashvili, A. Gogatishvili, V. Kokilashvili, M. Krbec, Weight Theory for Integral Transforms on Spaces of Homogeneous Type, Pitman Monographs and Surveys in Pure and Applied Mathematics, 92. Addison Wesley Longman, Harlow, 1998.

[16] I. Gohberg, N. Krupnik, One-Dimensional Linear Singular Integral Equations, Vols. 1, 2, Operator Theory: Advances and Applications, 53, 54. Birkhäuser Verlag, Basel, Boston, Berlin, 1992. Russian original: Shtiintsa, Kishinev, 1973.

[17] S. M. Grudsky, Singular integral equations and the Riemann boundary value problem with an infinite index in the space $L_{p}(\Gamma, \omega)$, Izv. Akad. Nauk SSSR Ser. Mat., 49 (1985), no. 1, 55-80 (in Russian). English translation: Math. USSR-Izv., 26 (1986), no. 1, 53-76.

[18] A. Kamińska, Indices, convexity and concavity in Musielak-Orlicz spaces, Funct. Approx. Comment. Math., 26 (1998), 67-84.

[19] A. Kamińska, B. Turett, Type and cotype in Musielak-Orlicz spaces. In: "Geometry of Banach spaces" (Strobl, 1989), pp. 165-180, London Math. Soc. Lecture Note Ser., 158. Cambridge Univ. Press, Cambridge, 1990.

[20] L. V. Kantorovich, G. P. Akilov, Functional Analysis, Nauka, Moscow, 3rd ed., 1984 (in Russian). English translation: Pergamon Press, Oxford, 2nd ed., 1982.

[21] N. Karapetiants, S. Samko, Equations with Involutive Operators, Birkhäuser Boston, Inc., Boston, MA, 2001.

[22] A. Yu. Karlovich, Algebras of singular integral operators with piecewise continuous coefficients on reflexive Orlicz spaces, Math. Nachr., 179 (1996), 187-222.

[23] A. Yu. Karlovich, Algebras of singular integral operators with piecewise continuous coefficients in rearrangement-invariant spaces with weight on Carleson curves. Ph. D. Thesis, Odessa, Ukraine, 1998, 142 p. (in Russian).

Available at http://www.math.ist.utl.pt/ akarlov/theses.html 
[24] A. Yu. Karlovich, Singular integral operators with piecewise continuous coefficients in reflexive rearrangement-invariant spaces, Integr. Equat. Oper. Theory, 32 (1998), 436-481.

[25] A. Yu. Karlovich, On the essential norm of the Cauchy singular integral operator in weighted rearrangement-invariant spaces, Integr. Equat. Oper. Theory, 38 (2000), 28-50.

[26] A. Yu. Karlovich, Algebras of singular integral operators with PC coefficients in rearrangement-invariant spaces with Muckenhoupt weights, J. Operator Theory, 47 (2002), 303-323.

[27] B. V. Khvedelidze, Linear discontinuous boundary problems in the theory of functions, singular integral equations and some of their applications, Akad. Nauk Gruzin. SSR. Trudy Tbiliss. Mat. Inst. Razmadze, 23 (1956), 3-158 (in Russian).

[28] B. V. Khvedelidze, The method of the Cauchy type integrals for discontinuous boundary value problems of the theory of holomorphic functions of one complex variable, Itogi nauki $i$ tehniki VINITI, Ser. Sovrem. probl. mat., 7 (1975), 5-162 (in Russian). English translation: J. Sov. Math., 7 (1977), 309-414.

[29] V. Kokilashvili, M. Krbec, Weighted Inequalities in Lorentz and Orlicz Spaces, World Scientific, New Jersey, London, Hong Kong, 1991.

[30] V. Kokilashvili, S. Samko, Singular integrals in weighted Lebesgue spaces with variable exponent, Departamento de Matemática, Instituto Superior Técnico, Lisboa, preprint 30/2002, December 2002.

[31] V. Kokilashvili, S. Samko, Singular integral equations in the Lebesgue spaces with variable exponent, Departamento de Matemática, Instituto Superior Técnico, Lisboa, preprint 2/2003, January 2003.

[32] O. Kováčik, J. Rákosník, On spaces $L^{p(x)}$ and $W^{k, p(x)}$, Czechoslovak Math. J., 41(116) (1991), no. 4, 592-618.

[33] M. A. Krasnoselskii, Ya. B. Rutickii, Convex Functions and Orlicz Spaces, Fizmatgiz, Moscow, 1958 (in Russian). English translation: Noordhoff Ltd., Groningen, 1961.

[34] M. Krbec, B. Opic, L. Pick, J. Rákosník, Some recent results on Hardy type operators in weighted function spaces and related topics. In: "Function spaces, differential operators and nonlinear analysis" (Friedrichroda, 1992), pp. 158-184, Teubner-Texte Math., 133. Teubner, Stuttgart, 1993

[35] S. G. Krein, Ju. I. Petunin, E. M. Semenov, Interpolation of Linear Operators, Nauka, Moscow, 1978 (in Russian). English translation: AMS Translations of Mathematical Monographs, 54. Providence, RI, 1982.

[36] N. Ya. Krupnik, Banach Algebras with Symbol and Singular Integral Operators, Operator Theory: Advances and Applications, 26. Birkhäuser Verlag, Basel, 1987.

[37] J. Lang, A. Nekvinda, L. Pick, Boundedness and compactness of general kernel integral operators from a weighted Banach function space into $L_{\infty}$, Department of Mathematical Analysis (KMA), Faculty of Mathematics and Physics, Charles University, Praha, Preprint MATHKMA-2003/94. Available at http://adela.karlin.mff.cuni.cz/ rokyta/preprint/

[38] G. S. Litvinchuk, I. M. Spitkovsky, Factorization of Measurable Matrix Functions, Operator Theory: Advances and Applications, 25. Birkhäuser Verlag, Basel, 1987.

[39] L. Maligranda, Indices and interpolation, Dissert. Math. (Rozprawy Mat.), 234 (1985), 1-49.

[40] S. G. Mikhlin, S. Prössdorf, Singular Integral Operators, Springer-Verlag, Berlin, 1986.

[41] J. Musielak, Orlicz Spaces and Modular Spaces, Lecture Notes in Mathematics, 1034. Springer-Verlag, Berlin, 1983.

[42] J. Musielak, W. Orlicz, On modular spaces, Studia Math., 18 (1959), 49-65.

[43] H. Nakano, Modulared Semi-Ordered Linear Spaces, Maruzen Co., Ltd., Tokyo, 1950.

[44] H. Nakano, Topology of Linear Topological Spaces, Maruzen Co., Ltd., Tokyo, 1951.

[45] W. Orlicz, Über konjugierte Exponentenfolgen, Studia Math., 3 (1931), 200-211. Reprinted in Wladyslaw Orlicz, "Collected Papers", PWN, Warsaw, 1988, pp. 200-213.

[46] L. Pick, M. Růžička, An example of a space $L^{p(x)}$ on which the Hardy-Littlewood maximal operator is not bounded, Expo. Math., 19 (2001), no. 4, 369-371.

[47] I. I. Privalov, Boundary Properties of Analytic Functions, Gosudarstv. Izdat. Tehn.-Teor. Lit., Moscow-Leningrad, 1950 (in Russian).

[48] M. Růžička, Electrorheological Fluids: Modeling and Mathematical Theory, Lecture Notes in Mathematics, 1748. Springer-Verlag, Berlin, 2000.

[49] I. B. Simonenko, The Riemann boundary value problem for $n$ pairs functions with measurable coefficients and its application to the investigation of singular integral operators in the spaces $L^{p}$ with weight, Izv. AN SSSR, Ser. Matem., 28 (1964), no. 2, 277-306 (in Russian). 
[50] I. B. Simonenko, A new general method of investigating linear operator equations of singular integral equation type, I-II, Izv. Akad. Nauk SSSR Ser. Mat., 29 (1965) 567-586 (Part I), 757-782 (Part II) (in Russian).

[51] I. B. Simonenko, Some general questions in the theory of the Riemann boundary value problem, Izv. AN SSSR, Ser. Matem., 32 (1968), no. 5, 1138-1146 (in Russian). English translation: Math. USSR Izv., 2 (1968), 1091-1099.

[52] I. Spitkovsky, Singular integral operators with $P C$ symbols on the spaces with general weights, J. Functional Analysis, 105 (1992), 129-143.

[53] M. Zippin, Interpolation of operators of weak type between rearrangement invariant spaces, J. Functional Analysis, 7 (1971), 267-284.

Departamento de Mathemática, Instituto Superior Técnico, Av. Rovisco Pais, 1049001 Lisboa, Portugal

E-mail address: akarlov@math.ist.utl.pt 